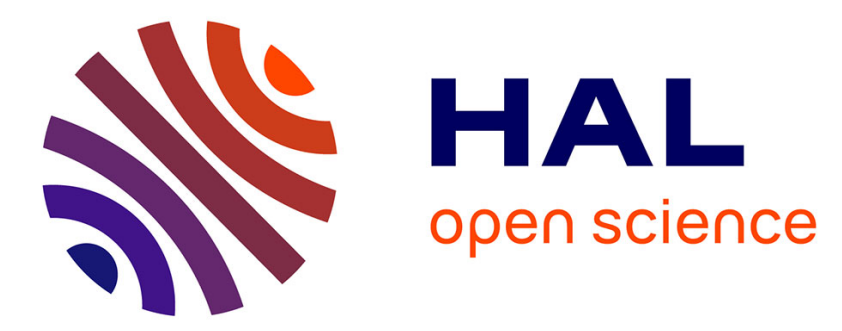

\title{
C-H Bond Alkylation of Cyclic Amides with Maleimides via a Site-Selective-Determining Six-Membered Ruthenacycle
}

Yu-Chao Yuan, Marion Goujon, Christian Bruneau, Thierry Roisnel, Rafael Gramage-Doria

\section{To cite this version:}

Yu-Chao Yuan, Marion Goujon, Christian Bruneau, Thierry Roisnel, Rafael Gramage-Doria. $\mathrm{C}-\mathrm{H}$ Bond Alkylation of Cyclic Amides with Maleimides via a Site-Selective-Determining SixMembered Ruthenacycle. Journal of Organic Chemistry, 2019, 84 (24), pp.16183-16191. 10.1021/acs.joc.9b02690 . hal-02442828

\section{HAL Id: hal-02442828 \\ https://hal.science/hal-02442828}

Submitted on 16 Jan 2020

HAL is a multi-disciplinary open access archive for the deposit and dissemination of scientific research documents, whether they are published or not. The documents may come from teaching and research institutions in France or abroad, or from public or private research centers.
L'archive ouverte pluridisciplinaire HAL, est destinée au dépôt et à la diffusion de documents scientifiques de niveau recherche, publiés ou non, émanant des établissements d'enseignement et de recherche français ou étrangers, des laboratoires publics ou privés. 
C-H Bond Alkylation of Cyclic Amides with Maleimides via a Site-Selective-Determining Six-Membered Ruthenacycle Yu-Chao Yuan, Marion Goujon, Christian Bruneau, Thierry Roisnel, and Rafael Gramage-Doria J. Org. Chem., Just Accepted Manuscript • Publication Date (Web): 25 Nov 2019

Downloaded from pubs.acs.org on November 25, 2019

\section{Just Accepted}

"Just Accepted" manuscripts have been peer-reviewed and accepted for publication. They are posted online prior to technical editing, formatting for publication and author proofing. The American Chemical Society provides "Just Accepted" as a service to the research community to expedite the dissemination of scientific material as soon as possible after acceptance. "Just Accepted" manuscripts appear in full in PDF format accompanied by an HTML abstract. "Just Accepted" manuscripts have been fully peer reviewed, but should not be considered the official version of record. They are citable by the Digital Object Identifier (DOI@). "Just Accepted" is an optional service offered to authors. Therefore, the "Just Accepted" Web site may not include all articles that will be published in the journal. After a manuscript is technically edited and formatted, it will be removed from the "Just Accepted" Web site and published as an ASAP article. Note that technical editing may introduce minor changes to the manuscript text and/or graphics which could affect content, and all legal disclaimers and ethical guidelines that apply to the journal pertain. ACS cannot be held responsible for errors or consequences arising from the use of information contained in these "Just Accepted" manuscripts. 


\title{
C-H Bond Alkylation of Cyclic Amides with Maleimides via a Site- Selective-Determining Six-Membered Ruthenacycle
}

\author{
Yu-Chao Yuan, Marion Goujon, Christian Bruneau, Thierry Roisnel, and Rafael Gramage-Doria* \\ Univ Rennes, CNRS, ISCR-UMR6226, F-35000 Rennes, France \\ Supporting Information
}

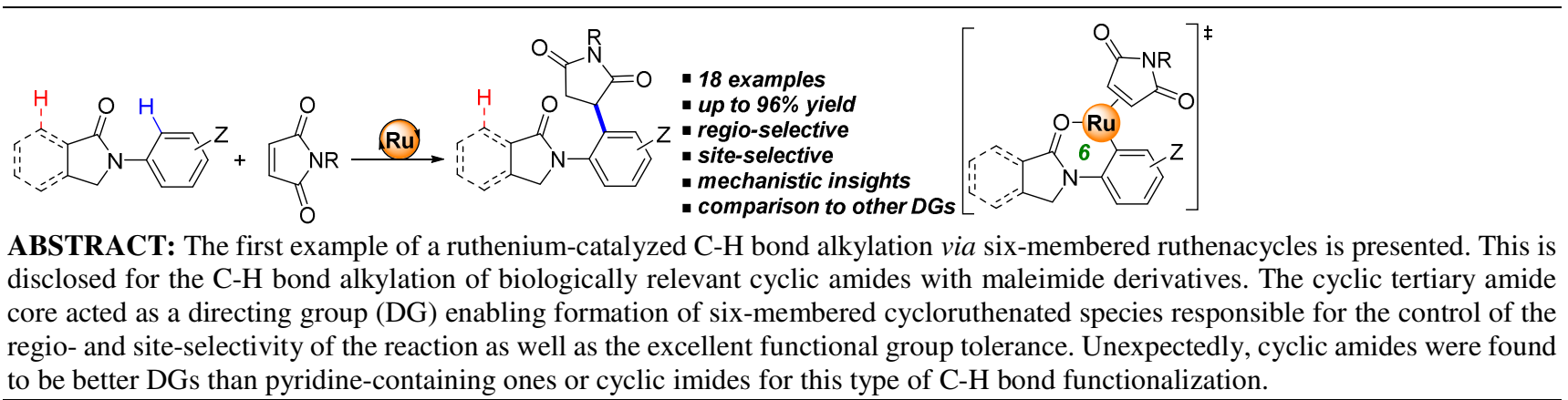

\section{INTRODUCTION}

Carbon-carbon bond-forming reactions via transition metal-catalyzed $\mathrm{C}-\mathrm{H}$ bond functionalization is an attractive methodology as it enables straightforward access to complex structures from low functionalized starting materials. ${ }^{1}$ It represents a sustainable entry to atom- and step-economy transformations relevant for academic and industrial laboratories. ${ }^{2}$ In this context, the use of directing groups to assist the $\mathrm{C}-\mathrm{H}$ activation step prior to the carbon-carbon bond formation is well recognized since the pioneering contribution from Murai and co-workers. ${ }^{3}$ As such, C$\mathrm{H}$ bond arylations, alkenylations and alkylations are nowadays employed using a wide range of transition metal catalysts. ${ }^{4}$

Amongst the different useful coupling partners enabling C$\mathrm{H}$ bond alkylations with transition metal catalysts, maleimides occupy a place of choice. ${ }^{\mathbf{5}, \mathbf{6}} \mathrm{On}$ one hand, their $\mathrm{C}=\mathrm{C}$ double bond is highly electron deficient which is important for coordinating to the cationic metal center during the catalysis, and in the other hand, it is a useful motif which enables straightforward post-functionalizations into succinimides, pyrrolidines, lactams and lactims. ${ }^{7}$ Importantly, maleimides are known to follow hydroarylation pathways instead of oxidative Heck ones during transition metal-catalyzed C-H bond functionalizations. The absence of a $\beta$-hydride elimination step results in a formal insertion of an alkyl fragment in the targeted $\mathrm{C}-\mathrm{H}$ bond. ${ }^{8}$ This feature has been explored in the $\mathrm{C}-\mathrm{H}$ bond alkylation of arenes with transition metal catalysts (Scheme 1). Different strong and weak directing groups have been disclosed to promote aromatic $\mathrm{C}-\mathrm{H}$ bond alkylations with maleimides in which five-membered metallacycle intermediates control the ortho-selectivity of the reaction as shown by Falck, Miura, Prabhu, Kim, Ackermann, Jeganmohan and others (Scheme 1A). ${ }^{5}$ Aromatic C-H bond alkylations via larger six-membered metallacycle intermediates had met success only for Rh-based catalysts (Scheme 1B). ${ }^{6}$ Strikingly, although ruthenium catalysts are known to accommodate six-membered ruthenacycle intermediates in aromatic $\mathrm{C}-\mathrm{H}$ bond arylations and alkenylations, ${ }^{9}$ the exploitation of such intermediates for $\mathrm{C}-\mathrm{H}$ bond alkylation is elusive to date. Herein, we report aromatic $\mathrm{C}-\mathrm{H}$ bond alkylations via six-membered ruthenacycles with maleimides as coupling partners. This is disclosed for the $\mathrm{C}-\mathrm{H}$ bond functionalization of isoindolinones, ${ }^{10} \mathrm{a}$ type of biologically relevant cyclic amides that, in addition, imposes selectivity issues as two $\mathrm{C}-\mathrm{H}$ sites could be accessible (Scheme 1C). 
Table 1. Optimization of the Ru-Catalyzed C-H Bond Alkylation of 1a with $N$-Methylmaleimide ${ }^{a}$ Catalyzed C-H Bond Alkylation of Arenes with Maleimides

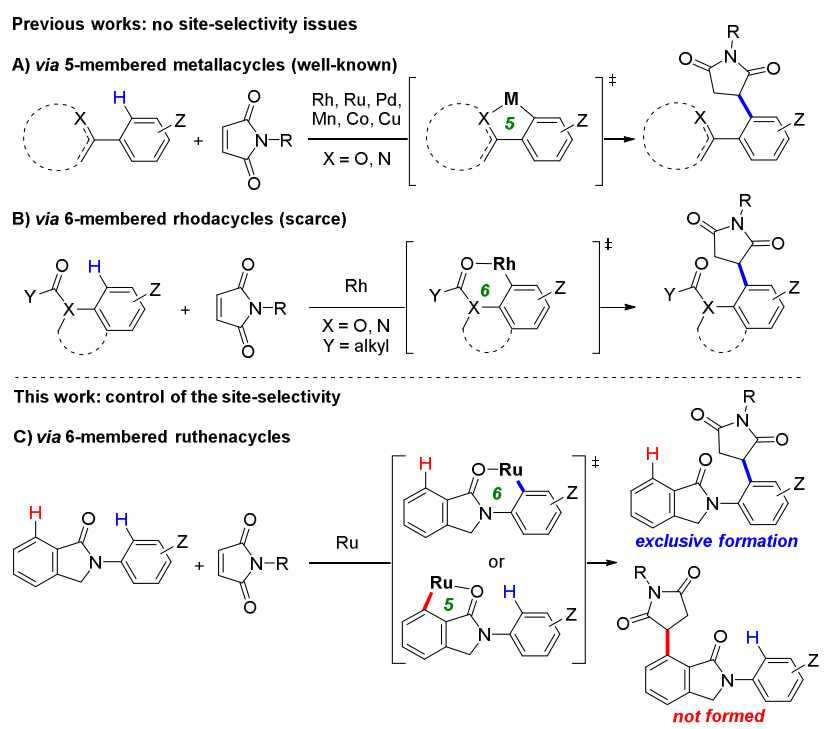

\section{- RESULTS AND DISCUSSION}

Initially, different reaction conditions were screened for the C$\mathrm{H}$ bond alkylation of $\mathrm{N}$-arylisoindolinone 1a with $\mathrm{N}$-methylmaleimide in the presence of a ruthenium catalyst (Table 1). The best reaction conditions we found consisted in $\left[\mathrm{RuCl}_{2}(p\right.$-cymene $)]_{2}(7.5 \mathrm{~mol} \%)$ as pre-catalyst, $\mathrm{AgSbF}_{6}(30 \mathrm{~mol} \%)$ as halide scavenger, $\mathrm{Cu}(\mathrm{OAc})_{2} \cdot 2 \mathrm{H}_{2} \mathrm{O}(1.5$ equiv) as an additive and $\mathrm{AcOH}$ (5 equiv) in 1,2-dichloroethane (DCE) as solvent at 120 ${ }^{\circ} \mathrm{C}$ for 18 hours. With these reaction conditions, $2 \mathbf{a}$ was obtained in $92 \%$ isolated yield with full conversion of $1 \mathbf{a}$ (Table 1, entry 1). The compound arising from the $\mathrm{C}-\mathrm{H}$ bond functionalization occuring in the aromatic ring $\mathrm{B}$ of $\mathbf{1 a}$ was not observed, and the alkenylated version of $\mathbf{2} \mathbf{a}$ formed only with trace amounts only detectable by GC-MS analysis. Control experiments indicated the need of all reagents (Table 1, entries 2-5) and the reaction in the presence of KOAc instead of $\mathrm{Cu}(\mathrm{OAc})_{2} \cdot \mathrm{H}_{2} \mathrm{O}$ led to trace amounts of product 2a (Table 1, entry 6). The presence of water and an excess of $\mathrm{AcOH}$, respectively, was detrimental for the catalysis (Table 1, entries 7-8). The reaction was finished after 6 hours (Table 1, entry 9), however, after 3 hours there was some starting material left (Table 1, entry 10). A recent aromatic $\mathrm{C}-\mathrm{H}$ bond alkylation lasting two hours with a very strong nitrogen-directing group (i.e. pyrimidine) via five-membered cobaltacycles has been reported. ${ }^{\mathbf{1 1}}$ We noted that the reaction was sensitive to temperature. For instance, at $100{ }^{\circ} \mathrm{C}$ 2a was formed in $90 \%$ yield (Table 1, entry 11 ), whereas at $80{ }^{\circ} \mathrm{C}$ no reaction was observed (Table 1 , entry 12 ). Lowering the loading of $\mathrm{N}$-methylmaleimide to 1.5 equivalents and $\left[\mathrm{RuCl}_{2}(p\right.$-cymene) $]_{2}$ to $5 \mathrm{~mol} \%$, respectively, still afforded the corresponding alkylated product $\mathbf{2 a}$ in $89 \%$ and $80 \%$ yield, respectively (Table 1, entries 13-14). Replacing the pre-catalyst $\left[\mathrm{RuCl}_{2}(p-\right.$ cymene) $]_{2}$ by first-row metal complexes ${ }^{12}$ such as $\mathrm{Mn}_{2}(\mathrm{CO})_{10}$ and $\mathrm{Co}(\mathrm{OAc})_{2} \cdot 4 \mathrm{H}_{2} \mathrm{O}$ afforded the starting materials completely unreacted (Table 1, entries 15-16). The optimal reaction conditions (Table 1, entry 1 ) were also applied to a larger scale experiment (50-fold increase of quantities), being the reaction equally efficient (see Experimental section below).

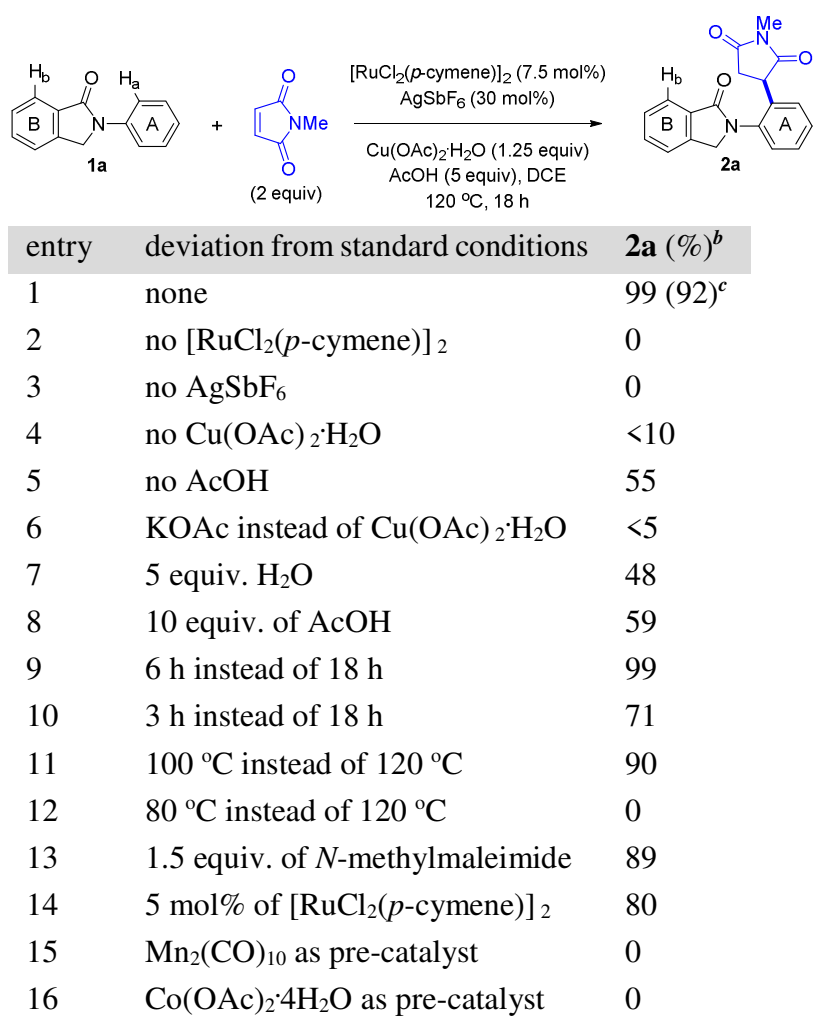

${ }^{a}$ Reaction conditions: 1a $(0.1 \mathrm{mmol}), \mathrm{N}$-methylmaleimide $(0.2 \mathrm{mmol})$, $\left[\mathrm{RuCl}_{2}(p \text {-cymene })\right]_{2}(7.5 \mathrm{~mol} \%), \mathrm{AgSbF}_{6}(30 \mathrm{~mol} \%), \mathrm{Cu}(\mathrm{OAc})_{2} \cdot \mathrm{H}_{2} \mathrm{O}(0.15$ $\mathrm{mmol}), \mathrm{AcOH}(0.5 \mathrm{mmol}), \mathrm{DCE}(0.5 \mathrm{~mL}), 120^{\circ} \mathrm{C}, 18 \mathrm{~h}$, argon. ${ }^{b}$ Yield of 2a determined by ${ }^{1} \mathrm{H}$ NMR analysis against an internal standard (dibromomethane). ${ }^{c}$ Isolated yield by column chromatography in brackets.

With the optimal reaction conditions in hand (Table 1, entry 1), we evaluated the scope of the catalysis with $\mathrm{N}$-arylisoindolinones (1) bearing various functional groups at different positions together with different $N$-substituted maleimides (Scheme 2). Arylisoindolinones substituted in para position of the $N$-phenyl ring with electronically different methyl, methoxy, ester, and chloro were tolerated by the catalysis affording the corresponding alkylated products $\mathbf{2 b - 2 e}$ in $70-96 \%$ isolated yields. The formation of $\mathbf{2 d}$ is a rare case in which the carboxylic acid ester group does not direct the transition metal-catalyzed $\mathrm{C}-\mathrm{H}$ bond alkylation using maleimides. ${ }^{13}$ Dioxolane-containing isoindolinone 2f, where the alkylation takes place at the most hindered ortho position, was obtained in $85 \%$ yield. Methyl-substituted isoindolinone in meta position of the $N$-phenyl ring afforded the alkylated products $2 \mathrm{~g}$ in $66 \%$ yield. In the case of $\mathbf{2 f}$ and $\mathbf{2 g}$, no formation of any other possible isomer was detected. On the other hand, the methoxy congener $\mathbf{2 h}$ was obtained in $76 \%$ yield with formation of traces of the other ortho-substituted isomer. The reaction was sensitive to the substitution pattern in the ortho position of the $N$-phenyl ring of the isoindolinones. For instance, the fluoro derivative $2 \mathbf{i}$ was obtained in an excellent $84 \%$ yield. However, swapping the fluoride group by a bulkier methyl group led to no reactivity. We assume that, as the bulkiness in the substitution in the ortho position increases, the $N$-phenyl axis rotation is restricted in such a way that it prevents formation of catalytically productive ruthenacycle intermediates. Maleimide derivatives containing different 
substitution patterns were also explored. For instance, 1a reacted with $N$-phenyl maleimide affording $\mathbf{2 j}$ in $78 \%$ yield and its molecular structure was unambiguously established by single crystal X-ray diffraction studies, which further supported the regio- and site-selectivity of the reaction. Fluoro-, bromo-, and nitro-containing maleimides were tolerated during the catalysis leading to the corresponding alkylated products $\mathbf{2 k - 2 m}$ in $64-78 \%$ yield. $N$-benzyl maleimide afforded the corresponding alkylated product $\mathbf{2 n}$ in $88 \%$ isolated yield. Analogously, the trifluoro-containing alkylated product 20 was obtained in a similar yield. Interestingly, heterocycle-containing maleimides such as thiophene were tolerated affording the alkylated product $2 \mathbf{p}$ in $58 \%$ yield. The reactions were very selective as only the product 2 formed, with trace amounts of the remaining reagents. Limitations appeared when using unsubstituted $N$-H maleimide as coupling partner. In this case, the desired product $\mathbf{2 q}$ was observed in $10 \%$ yield together with unreacted starting materials. We also noted that nitrile and iodide substitution patterns were not compatible, and maleic anhydride and dimethyl maleate were unreactive as well (Scheme 2). Notably, only mono-functionalized products $\mathbf{2 a - 2 r}$ were observed during the catalysis with no evidences for bis-functionalization.

Scheme 2. Evaluation of the Scope for the Ru-Catalyzed CH Bond Alkylation of 1 with $N$-substituted Maleimides ${ }^{a, b}$

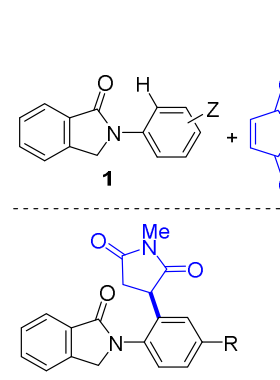

2b: $\mathrm{R}=\mathrm{Me}, 88 \%$ 2d: $\mathrm{R}=\mathrm{CO}_{2} \mathrm{Et}, 70 \%$ 2e: $\mathrm{R}=\mathrm{Cl}, 96 \%$ $\mathrm{R}=\mathrm{CN}, 0 \%$

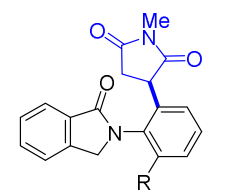

2i: $R=F, 84 \%$

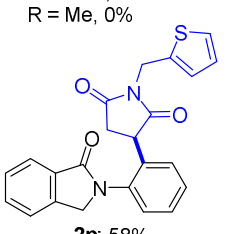

2p: $58 \%$ 2c: $\mathrm{R}=\mathrm{OMe}, 91 \%$
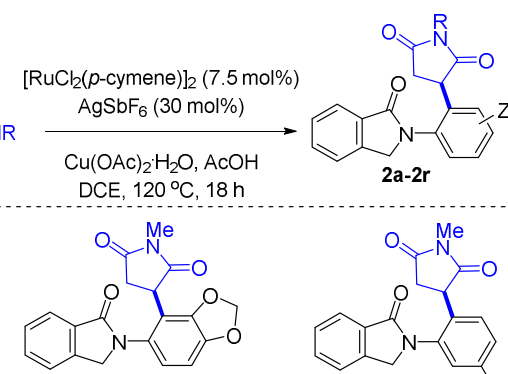

2f: $85 \%$
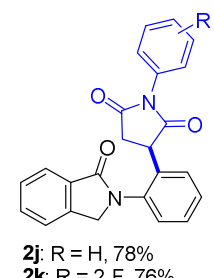
2l: $R=2-\mathrm{Br}, 64 \%$ 2m: $\mathrm{R}=4-\mathrm{NO}_{2}, 70 \%$ $\mathrm{R}=4-\mathrm{I}, 0 \%$

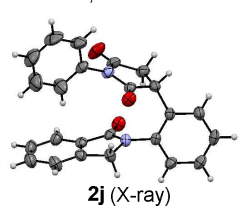

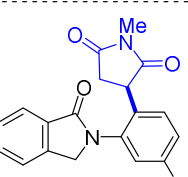
2g: $R=M e, 66 \% R$

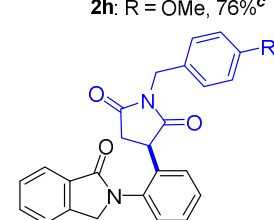

2n: $\mathrm{R}=\mathrm{H}, 88 \%$ 2o: $\mathrm{R}=\mathrm{CF}_{3}, 88 \%$

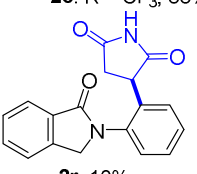

2r: $10 \%$

Reluctant coupling partners

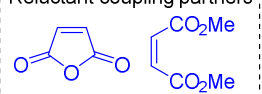
2h: $\mathrm{R}=\mathrm{OMe}, 76 \%^{\mathrm{c}}$

${ }^{a}$ Reaction conditions: $1(0.5 \mathrm{mmol}), N$-substituted maleimide $(1 \mathrm{mmol})$, $\left[\mathrm{RuCl}_{2}(p \text {-cymene })\right]_{2}(5 \mathrm{~mol} \%), \mathrm{AgSbF}_{6}(30 \mathrm{~mol} \%), \mathrm{Cu}(\mathrm{OAc})_{2} \cdot \mathrm{H}_{2} \mathrm{O}(0.75$ $\mathrm{mmol})$, AcOH (2.5 mmol), DCE $(2.5 \mathrm{~mL}), 120{ }^{\circ} \mathrm{C}, 18 \mathrm{~h}$, argon. ${ }^{b}$ Isolated yield by column chromatography. ${ }^{c}$ The minor regioisomer is not depicted (see details in the Supporting Information).

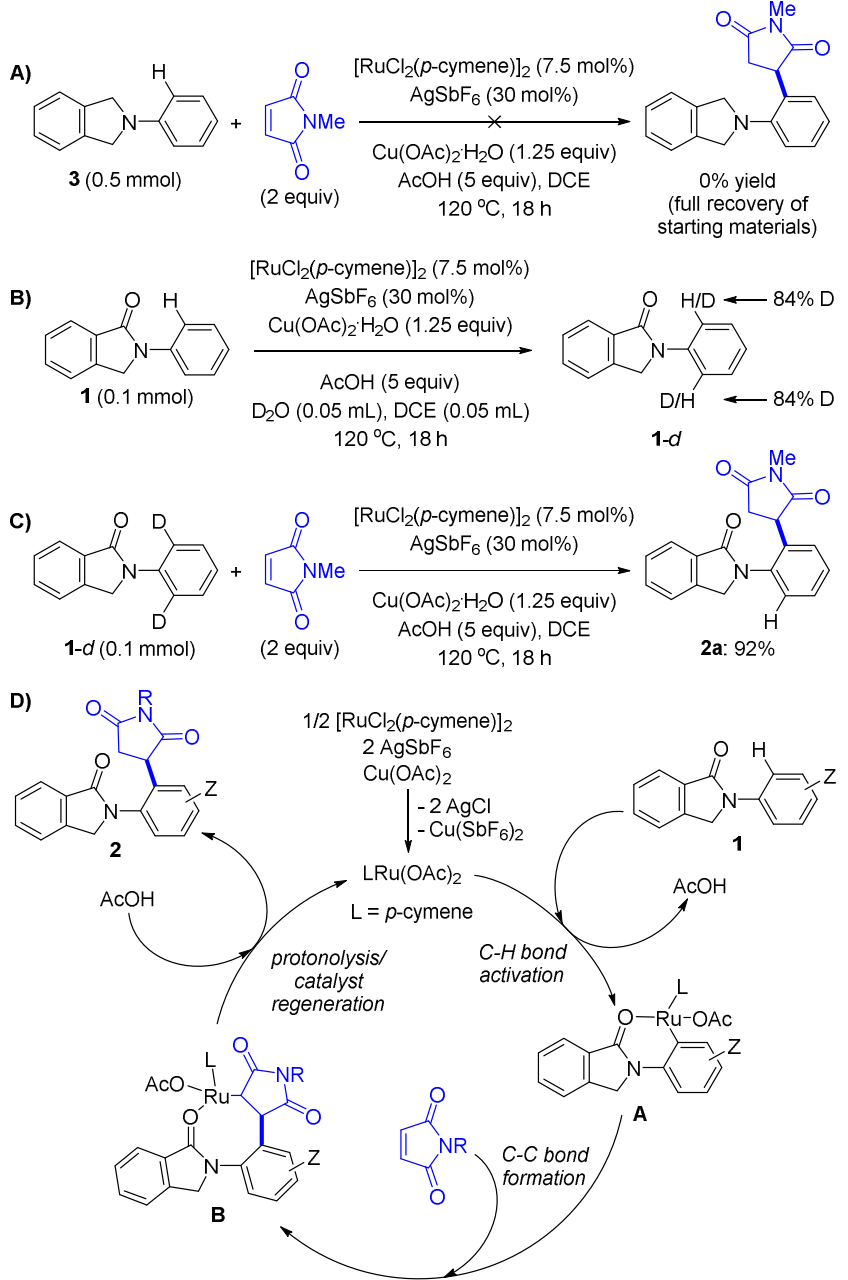

Figure 1. Mechanistic considerations.

In order to gain some insights about the reaction mechanism, we performed a reaction with a substrate lacking any carbonyl group (3). Under the standard reaction conditions, no alkylated product was formed (Figure 1A), thus indicating the potential role of the carbonyl group in $\mathbf{1}$ as a weak directing group in the $\mathrm{C}-\mathrm{H}$ bond activation step. ${ }^{14}$ Additionally, deuteration experiments with 1a afforded the product 1a- $d$ with $84 \%$ deuterium incorporation in the protons that underwent subsequent alkylation in the catalysis (Figure 1B), with no other deuteration occurring in any other protons of the molecule. When 1- $d$ was used as a substrate for the C-H bond alkylation with $\mathrm{N}$ methylmaleimide under standard reaction conditions, the expected product 2a was obtained with no deuterium on it (Figure 1C), suggesting the participation of acetic acid in the last step of the catalysis and the reversibility of the $\mathrm{C}-\mathrm{H}$ bond activation step. These data together with precedents in the literature, ${ }^{\mathbf{5 , 1 5}}$ suggested the plausible, simplified mechanism displayed in Figure 1D. First, chloride-free ruthenium species may form, which, after a concerted metalation-deprotonation step with $\mathbf{1}$ leads to the six-membered ruthenacycle $\mathbf{A}$ with formation of acetic acid. Coordination of maleimide to ruthenium followed by alkene insertion leads to $\mathbf{B}$, which, after protonolysis with acetic acid enables the formation of $\mathbf{2}$ and regeneration of the catalytically active ruthenium species. The need for over-stoichiometric amounts of acetic acid and $\mathrm{Cu}(\mathrm{OAc})_{2} \cdot \mathrm{H}_{2} \mathrm{O}$ in the catalysis might 
be a consequence of the low kinetics ascribed to this transformation and/or solubility issues. It is noteworthy that with maleimide substrates, the formation of an intermediate of type $\mathbf{B}$ is not favorable for $\beta$-hydride elimination ${ }^{\mathbf{8}}$ as observed in the case of alkenylation with acyclic olefins under related conditions, ${ }^{\mathbf{1 6 b}}$ but prone to be cleaved by protonolysis even in the absence of added acetic acid (Table 1 , entry 5 ).

For comparison purposes, other directing groups potentially enabling six-membered ruthenacycle intermediates were explored for this aromatic $\mathrm{C}-\mathrm{H}$ bond alkylation reaction (Scheme 3). Cyclic imides such as $\mathbf{4}$ and $\mathbf{5}$, which are known to enable ruthenium-catalyzed $\mathrm{C}-\mathrm{H}$ bond hydroxylations and alkenylations to some extent, ${ }^{\mathbf{1 6}}$ did not react under the standard reaction conditions for $\mathrm{C}-\mathrm{H}$ bond alkylation. Likely, a combination of steric and electronic effects prevents the formation of six-membered ruthenacycles for cyclic imides under these reaction conditions as it has been recently reported for ruthenium-catalyzed $\mathrm{C}-\mathrm{H}$ bond arylations with the same substrates. ${ }^{\mathbf{1 6 c}}$ On the other hand, pyrrolidinone $\mathbf{6}$, which promotes $\mathrm{C}-\mathrm{H}$ bond alkenylations and benzoxylations, ${ }^{91,17}$ smoothly reacted leading to the alkylated product in $80 \%$ yield with the same regio- and site-selectivity as isoindolinones $\mathbf{1}$ and no evidences for bis-functionalization. Surprisingly, oxazolidinone 7, which is structurally related to $\mathbf{6}$ and it is known to efficiently promote $\mathrm{C}-\mathrm{H}$ bond alkenylations, ${ }^{91}$ did not react under our standard reaction conditions for $\mathrm{C}-\mathrm{H}$ bond alkylation. Nitrogen-containing directing groups such as benzylpyridine $\mathbf{8}$ and benzoylpyridine $\mathbf{9}$, the latter being known to promote $\mathrm{C}-\mathrm{H}$ bond arylations, ${ }^{\mathbf{9 c}, \mathbf{e}}$ did not afford any alkylated products in our hands. Consequently, although nitrogen-containing directing groups are largely considered as better directing groups than ketones, the presented ruthenium-catalyzed C-H bond alkylation methodology emphasizes that it is not always the case, and a balance between both coordination strength and stereo-electronic effects need to be considered.

Scheme 3. Evaluation of Different Directing Groups in the Ru-Catalyzed C-H Bond Alkylation with Maleimides
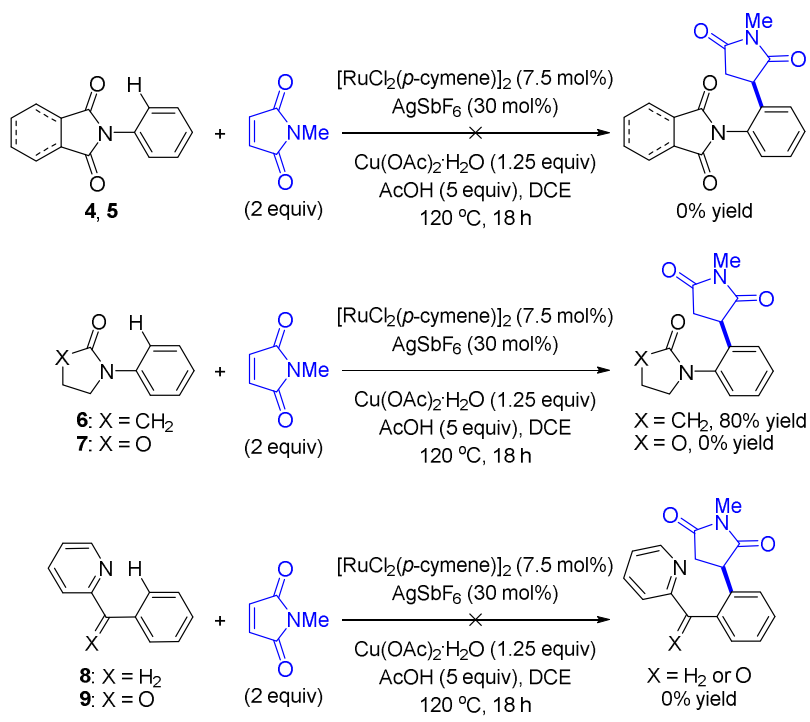

- CONCLUSIONS

In summary, we have shown that ruthenium catalysts enabled the regio-, mono-, and site-selective alkylation of $N$-arylisoindolinones with $N$-substituted maleimides in high yields and an excellent functional group tolerance [alkyl, benzyl, aryl, (a)cyclic ether, carboxylic ester, nitro, halides (i.e. $\mathrm{F}, \mathrm{Cl}, \mathrm{Br}, \mathrm{CF}_{3}$ ), heteroaromatics (i.e. thiophene)]. A combination of control reactions and deuteration experiments support that the catalysis occurs via a reversible and large six-membered ruthenacycle in the key $\mathrm{C}-\mathrm{H}$ bond activation step, which is unprecedented for ruthenium catalysts in $\mathrm{C}-\mathrm{H}$ bond alkylation reactions. The potential of isoindolinones enabling $\mathrm{C}-\mathrm{H}$ bond functionalizations arises from the capability of the cyclic tertiary amide to behave as a weak directing group throughout the catalysis. It is also demonstrated that cyclic amides (i.e. isoindolinones and pyrrolidinones), unexpectedly, outperform cyclic imides, oxazolidinones or, even, pyridine-containing directing groups in ruthenium-catalyzed $\mathrm{C}-\mathrm{H}$ bond alkylations when six-membered intermediates are formed. Overall, this study highlights the importance of the balance between the coordination strength and the stereoelectronic properties for directing groups to be efficient.

\section{- EXPERIMENTAL SECTION}

General Methods. All reagents were obtained from commercial sources and used as supplied. All reactions were carried out in flame-dried glassware under argon atmosphere unless otherwise noted. Catalytic experiments were performed in Schlenktype flasks under argon atmosphere unless otherwise noted. Organic solutions were concentrated under reduced pressure using a rotary evaporator. Thin-layer chromatography (TLC) were carried out on $0.25 \mathrm{~mm}$ Merck silica gel (60-F254). Flash column chromatography was performed using silica gel Silica 60 M, 0.04-0.063 mm. Technical grade petroleum ether (40-60), nheptane and ethyl acetate were used for column chromatography. $\mathrm{CDCl}_{3}$ was stored under nitrogen over molecular sieves. NMR spectra were recorded on an AVANCE III 400 spectrometer. ${ }^{1} \mathrm{H}$ NMR spectra were referenced to residual protiated solvent ( $\delta=7.26 \mathrm{ppm}$ for $\mathrm{CDCl}_{3}$ and $\delta=2.05 \mathrm{ppm}$ for acetone- $d_{6}$ ) and ${ }^{13} \mathrm{C}$ chemical shifts are reported relative to deuterated solvents $\left(\delta=77.0 \mathrm{ppm}\right.$ for $\mathrm{CDCl}_{3}$ and $\delta=29.8 \mathrm{ppm}$ for acetone$d_{6}$ ) [Note: acetone- $d_{6}$ contains traces of water at $c a .3 \mathrm{ppm}$ ]. The peak patterns are indicated as follows: s, singlet; d, doublet; $t$, triplet; q, quartet; m, multiplet, and br. for broad. GC-MS analyses were performed with a GCMS-QP2010S (Shimadzu) instrument with a GC-2010 equipped with a 30 m capillary column (Supelco, SLBTM-5ms, fused silica capillary column, 30 $\mathrm{m} \times 0.25 \mathrm{~mm} \times 0.25 \mathrm{~mm}$ film thickness), which was used with helium as the vector gas. The following GC conditions were used: initial temperature $80{ }^{\circ} \mathrm{C}$ for 2 minutes, then rate 20 ${ }^{\circ} \mathrm{C} /$ min until $280{ }^{\circ} \mathrm{C}$ and $280{ }^{\circ} \mathrm{C}$ for 28 minutes. HRMS were recorded on a Waters Q-Tof 2 mass spectrometer at the corresponding facilities of the CRMPO, Centre Régional de Mesures Physiques de l'Ouest, Université de Rennes 1 . Compounds $\mathbf{1},{ }^{\mathbf{1 8}}$ $3,{ }^{19} 4^{20} 5^{21}$ and $7^{91}$ have been synthesized according to literature protocols.

General Procedure for the Ruthenium-Catalyzed C-H bond Alkylation Reactions, and Characterization of Products: $\left[\mathrm{RuCl}_{2} \text { (p-cymene) }\right]_{2}(7.5 \mathrm{~mol} \%, 22.9 \mathrm{mg}), \mathrm{AgSbF}_{6}(30 \mathrm{~mol} \%$, $51.5 \mathrm{mg}), \mathrm{Cu}(\mathrm{OAc})_{2} \cdot \mathrm{H}_{2} \mathrm{O}(0.75 \mathrm{mmol}, 149.7 \mathrm{mg}, 1.5$ equiv. $)$, the corresponding substrate $1(0.5 \mathrm{mmol}, 1.0$ equiv. $)$, and the corresponding maleimide derivative ( $1 \mathrm{mmol}, 2.0$ equiv.) were taken in a $15 \mathrm{~mL}$ pressure tube, which was equipped with a 
magnetic stirrer. To this mixture was added AcOH (2.5 mmol, $0.14 \mathrm{~mL}, 5.0$ equiv.) and solvent DCE $(2.5 \mathrm{~mL})$ under Argon. Then the reaction vial was sealed with the screw cap and allowed to stir at $120^{\circ} \mathrm{C}$ in an oil bath for $18 \mathrm{~h}$. After being cooled to ambient temperature, the reaction mixture was diluted with $\mathrm{CH}_{2} \mathrm{Cl}_{2}$ and then filtered through Celite. After evaporation of the solvent in vacuo, the crude product was purified by column chromatography on silica gel ( $n$-heptane/EtOAc: 10:1 to 1:2) to give the desired product 2.

1-Methyl-3-(2-(1-oxoisoindolin-2-yl)phenyl)pyrrolidine-2,5dione $(\mathbf{2 a})$. Brown solid, yield $=92 \%, 147.4 \mathrm{mg}$. Mp: 162-164 ${ }^{\circ} \mathrm{C} .{ }^{1} \mathrm{H} \mathrm{NMR}\left(400 \mathrm{MHz}, \mathrm{CDCl}_{3}\right): \delta=7.84(\mathrm{~d}, J=7.6 \mathrm{~Hz}, 1 \mathrm{H})$, $7.58(\mathrm{dd}, J=7.6,7.2 \mathrm{~Hz}, 1 \mathrm{H}), 7.50-7.46(\mathrm{~m}, 2 \mathrm{H}), 7.40-7.34(\mathrm{~m}$, $2 \mathrm{H}), 7.28-7.25(\mathrm{~m}, 1 \mathrm{H}), 7.22-7.20(\mathrm{~m}, 1 \mathrm{H}), 4.85(\mathrm{~d}, J=17.2 \mathrm{~Hz}$, $1 \mathrm{H}), 4.77(\mathrm{~d}, J=17.2 \mathrm{~Hz}, 1 \mathrm{H}), 4.10(\mathrm{dd}, J=9.6,5.2 \mathrm{~Hz}, 1 \mathrm{H})$, $3.15(\mathrm{dd}, J=18.8,9.6 \mathrm{~Hz}, 1 \mathrm{H}), 2.97(\mathrm{dd}, J=18.4,5.2 \mathrm{~Hz}, 1 \mathrm{H})$, $2.76(\mathrm{~s}, 3 \mathrm{H}) \mathrm{ppm} .{ }^{13} \mathrm{C}\left\{{ }^{1} \mathrm{H}\right\}$ NMR $\left(100 \mathrm{MHz}, \mathrm{CDCl}_{3}\right): \delta=177.9$, 176.3, 168.4, 141.9, 137.3, 136.7, 132.1, 131.4, 129.1, 129.0, 128.5, 128.4, 124.1, 122.9, 54.1, 42.4, 36.8, 24.8 ppm. HRMS (ESI) calcd. for $[\mathrm{M}+\mathrm{Na}]^{+} \mathrm{C}_{19} \mathrm{H}_{16} \mathrm{~N}_{2} \mathrm{O}_{3} \mathrm{Na} 343.1053$, found 343.1056 (1 ppm).

1-Methyl-3-(5-methyl-2-(1-oxoisoindolin-2-yl)phenyl)pyrrolidine-2,5-dione $(\mathbf{2 b})$. Brown solid, yield $=88 \%, 148.0 \mathrm{mg}$. Mp: $<50{ }^{\circ} \mathrm{C} .{ }^{1} \mathrm{H}$ NMR $\left(400 \mathrm{MHz}, \mathrm{CDCl}_{3}\right): \delta=7.83(\mathrm{~d}, J=8.0 \mathrm{~Hz}$, $1 \mathrm{H}), 7.56(\mathrm{ddd}, J=8.0,8.0,1.2 \mathrm{~Hz}, 1 \mathrm{H}), 7.47(\mathrm{dd}, J=6.8,6.0$ $\mathrm{Hz}, 2 \mathrm{H}), 7.19-7.13(\mathrm{~m}, 2 \mathrm{H}), 7.00(\mathrm{~s}, 1 \mathrm{H}), 4.82(\mathrm{~d}, J=17.2 \mathrm{~Hz}$, $1 \mathrm{H}), 4.73(\mathrm{~d}, J=17.2 \mathrm{~Hz}, 1 \mathrm{H}), 4.05(\mathrm{dd}, J=9.2,5.2 \mathrm{~Hz}, 1 \mathrm{H})$, $3.13(\mathrm{dd}, J=18.8,9.6 \mathrm{~Hz}, 1 \mathrm{H}), 2.97(\mathrm{dd}, J=18.8,4.8 \mathrm{~Hz}, 1 \mathrm{H})$, 2.77 (s, 3H), $2.34(\mathrm{~s}, 3 \mathrm{H}) \mathrm{ppm} .{ }^{13} \mathrm{C}\left\{{ }^{1} \mathrm{H}\right\}$ NMR $(100 \mathrm{MHz}$, $\left.\mathrm{CDCl}_{3}\right): \delta=178.0,176.3,168.5,141.9,139.1,136.3,134.5$, $132.0,131.5,129.8,128.7,128.3,128.2,124.0,122.9,54.1$, $42.3,36.8,24.8,21.1 \mathrm{ppm}$. HRMS (ESI) calcd. for $[\mathrm{M}+\mathrm{Na}]^{+}$ $\mathrm{C}_{20} \mathrm{H}_{18} \mathrm{~N}_{2} \mathrm{O}_{3} \mathrm{Na} 357.1209$, found 357.1209 (0 ppm).

3-(5-Methoxy-2-(1-oxoisoindolin-2-yl)phenyl)-1-methylpyrrolidine-2,5-dione $(2 \mathrm{c})$. White solid, yield $=91 \%, 160.0 \mathrm{mg}$. Mp: 167-169 ${ }^{\circ} \mathrm{C} .{ }^{1} \mathrm{H}$ NMR $\left(400 \mathrm{MHz}, \mathrm{CDCl}_{3}\right): \delta=7.78(\mathrm{~d}, J=7.6$ $\mathrm{Hz}, 1 \mathrm{H}), 7.53$ (dd, $J=7.6,7.2 \mathrm{~Hz}, 1 \mathrm{H}), 7.45-7.41(\mathrm{~m}, 2 \mathrm{H}), 7.15$ $(\mathrm{d}, J=8.8 \mathrm{~Hz}, 1 \mathrm{H}), 6.86(\mathrm{dd}, J=8.8,2.8 \mathrm{~Hz}, 1 \mathrm{H}), 6.69(\mathrm{~s}, 1 \mathrm{H})$, $4.76(\mathrm{~d}, J=17.6 \mathrm{~Hz}, 1 \mathrm{H}), 4.67(\mathrm{~d}, J=17.2 \mathrm{~Hz}, 1 \mathrm{H}), 4.00(\mathrm{dd}, J$ $=9.2,5.6 \mathrm{~Hz}, 1 \mathrm{H}), 3.74(\mathrm{~s}, 3 \mathrm{H}), 3.07(\mathrm{dd}, J=18.8,9.6 \mathrm{~Hz}, 1 \mathrm{H})$, $2.94(\mathrm{dd}, J=18.8,4.8 \mathrm{~Hz}, 1 \mathrm{H}), 2.70(\mathrm{~s}, 3 \mathrm{H}) \mathrm{ppm} .{ }^{13} \mathrm{C}\left\{{ }^{1} \mathrm{H}\right\} \mathrm{NMR}$ $\left(100 \mathrm{MHz}, \mathrm{CDCl}_{3}\right): \delta=177.7,176.1,168.6,159.6,141.9$, $137.8,131.9,131.4,129.6,128.2,123.9,122.8,113.8,55.5$, 54.2, 42.5, 36.6, $24.7 \mathrm{ppm}$. HRMS (ESI) calcd. for $[\mathrm{M}+\mathrm{Na}]^{+}$ $\mathrm{C}_{20} \mathrm{H}_{18} \mathrm{~N}_{2} \mathrm{O}_{4} \mathrm{Na} 373.1159$, found 373.1158 (0 ppm).

Ethyl 3-(1-methyl-2,5-dioxopyrrolidin-3-yl)-4-(1-oxoisoindolin-2-yl)benzoate (2d). Yellow solid, yield $=70 \%, 136.9 \mathrm{mg}$. Mp: $<50{ }^{\circ} \mathrm{C} .{ }^{1} \mathrm{H}$ NMR $\left(400 \mathrm{MHz}, \mathrm{CDCl}_{3}\right): \delta=7.97(\mathrm{dd}, J=8.4$, $2.0 \mathrm{~Hz}, 1 \mathrm{H}), 7.86(\mathrm{~d}, J=2.0 \mathrm{~Hz}, 1 \mathrm{H}), 7.77(\mathrm{~d}, J=7.6 \mathrm{~Hz}, 1 \mathrm{H})$, $7.54(\mathrm{dd}, J=7.6,6.8 \mathrm{~Hz}, 1 \mathrm{H}), 7.43(\mathrm{dd}, J=7.6,7.2 \mathrm{~Hz}, 2 \mathrm{H})$, $7.32(\mathrm{~d}, J=8.4 \mathrm{~Hz}, 1 \mathrm{H}), 4.79(\mathrm{~d}, J=16.8 \mathrm{~Hz}, 2 \mathrm{H}), 4.31(\mathrm{q}, J=$ $7.2 \mathrm{~Hz}, 2 \mathrm{H}), 4.14(\mathrm{dd}, J=9.6,5.2 \mathrm{~Hz}, 1 \mathrm{H}), 3.15(\mathrm{dd}, J=18.8$, $9.6 \mathrm{~Hz}, 1 \mathrm{H}), 2.98(\mathrm{dd}, J=18.4,5.6 \mathrm{~Hz}, 1 \mathrm{H}), 2.71(\mathrm{~s}, 3 \mathrm{H}), 1.32$ (t, $J=7.2 \mathrm{~Hz}, 3 \mathrm{H}) \mathrm{ppm} .{ }^{13} \mathrm{C}\left\{{ }^{1} \mathrm{H}\right\} \mathrm{NMR}\left(100 \mathrm{MHz}, \mathrm{CDCl}_{3}\right): \delta=$ $177.4,175.9,168.1,165.1,141.8,141.4,136.7,132.2,131.0$, 130.7, 129.9, 129.7, 128.4, 128.2, 124.0, 122.9, 61.3, 53.7, 42.4, $36.4,24.8,14.2 \mathrm{ppm}$. HRMS (ESI) calcd. for $[\mathrm{M}+\mathrm{Na}]^{+}$ $\mathrm{C}_{22} \mathrm{H}_{20} \mathrm{~N}_{2} \mathrm{O}_{5} \mathrm{Na} 415.1264$, found 415.1260 (1 ppm).

3-(5-Chloro-2-(1-oxoisoindolin-2-yl)phenyl)-1-methylpyrrolidine-2,5-dione $(2 e)$. White solid, yield $=96 \%, 170.0 \mathrm{mg}$. Mp: 192-194 ${ }^{\circ} \mathrm{C} .{ }^{1} \mathrm{H}$ NMR $\left(400 \mathrm{MHz}, \mathrm{CDCl}_{3}\right): \delta=7.87$ (d, $J=7.2$
$\mathrm{Hz}, 1 \mathrm{H}), 7.62(\mathrm{ddd}, J=7.6,7.6,1.2 \mathrm{~Hz}, 1 \mathrm{H}), 7.52(\mathrm{dd}, J=7.6$, $7.2 \mathrm{~Hz}, 2 \mathrm{H}), 7.38(\mathrm{dd}, J=8.4,2.4 \mathrm{~Hz}, 1 \mathrm{H}), 7.24(\mathrm{~d}, J=8.4 \mathrm{~Hz}$, $2 \mathrm{H}), 4.86(\mathrm{~d}, J=17.2 \mathrm{~Hz}, 1 \mathrm{H}), 4.78(\mathrm{~d}, J=16.8 \mathrm{~Hz}, 1 \mathrm{H}), 4.10$ (dd, $J=9.6,5.2 \mathrm{~Hz}, 1 \mathrm{H}), 3.20(\mathrm{dd}, J=18.4,9.6 \mathrm{~Hz}, 1 \mathrm{H}), 3.01$ (dd, $J=18.4,5.2 \mathrm{~Hz}, 1 \mathrm{H}), 2.82(\mathrm{~s}, 3 \mathrm{H}) \mathrm{ppm} .{ }^{13} \mathrm{C}\left\{{ }^{1} \mathrm{H}\right\} \mathrm{NMR}$ $\left(100 \mathrm{MHz}, \mathrm{CDCl}_{3}\right): \delta=177.4,175.9,168.6,141.9,138.5$, 136.0, 134.8, 132.4, 131.2, 129.8, 129.4, 128.6, 124.4, 123.1, 54.1, 42.5, 36.6, $25.1 \mathrm{ppm}$. HRMS (ESI) calcd. for $[\mathrm{M}+\mathrm{Na}]^{+}$ $\mathrm{C}_{19} \mathrm{H}_{15} \mathrm{~N}_{2} \mathrm{O}_{3}{ }^{35} \mathrm{ClNa}$ 377.0663, found 377.0663 (0 ppm).

1-Methyl-3-(5-(1-oxoisoindolin-2-yl)benzo[d][1,3]dioxol-4$y$ l)pyrrolidine-2,5-dione $(2 f)$. Brown solid, yield $=85 \%, 155.0$ mg. $\mathrm{Mp}>250{ }^{\circ} \mathrm{C}$ dec. ${ }^{1} \mathrm{H}$ NMR $\left(400 \mathrm{MHz}, \mathrm{CDCl}_{3}\right): \delta=7.88$ $(\mathrm{d}, J=7.6 \mathrm{~Hz}, 1 \mathrm{H}), 7.60(\mathrm{dd}, J=7.6,7.2 \mathrm{~Hz}, 1 \mathrm{H}), 7.51(\mathrm{dd}, J=$ 8.0, 8.0 Hz, 2H), 6.85-6.78 (m, 2H), $6.00(\mathrm{~s}, 2 \mathrm{H}), 4.79(\mathrm{~d}, J=$ $17.6 \mathrm{~Hz}, 2 \mathrm{H}), 3.95(\mathrm{dd}, J=8.8,7.2 \mathrm{~Hz}, 1 \mathrm{H}), 3.20-3.08(\mathrm{~m}, 2 \mathrm{H})$, $2.85(\mathrm{~s}, 3 \mathrm{H}) \mathrm{ppm} .{ }^{13} \mathrm{C}\left\{{ }^{1} \mathrm{H}\right\} \mathrm{NMR}\left(100 \mathrm{MHz}, \mathrm{CDCl}_{3}\right): \delta=177.5$, $176.5,168.8,147.5,141.9,132.2,131.6,131.2,128.5,124.4$, 123.0, 121.5, 118.7, 108.4, 102.3, 54.6, 38.9, 35.1, 25.0 ppm. HRMS (ESI) calcd. for $[\mathrm{M}+\mathrm{Na}]^{+} \mathrm{C}_{20} \mathrm{H}_{16} \mathrm{~N}_{2} \mathrm{O}_{5} \mathrm{Na} 387.0951$, found 387.0949 (1 ppm).

1-Methyl-3-(4-methyl-2-(1-oxoisoindolin-2-yl)phenyl)pyrrolidine-2,5-dione (2g). White solid, yield $=66 \%, 109.9 \mathrm{mg}$. Mp: 153-155 ${ }^{\circ} \mathrm{C}$. ${ }^{1} \mathrm{H}$ NMR $\left(400 \mathrm{MHz}, \mathrm{CDCl}_{3}\right): \delta=7.85(\mathrm{~d}, J=8.4$ $\mathrm{Hz}, 1 \mathrm{H}), 7.58(\mathrm{dd}, J=7.2,7.2 \mathrm{~Hz}, 1 \mathrm{H}), 7.49(\mathrm{dd}, J=6.8,6.4$ $\mathrm{Hz}, 2 \mathrm{H}), 7.18$ (d, $J=7.6 \mathrm{~Hz}, 1 \mathrm{H}), 7.10$ (d, $J=6.4 \mathrm{~Hz}, 2 \mathrm{H}), 4.86$ $(\mathrm{d}, J=17.2 \mathrm{~Hz}, 1 \mathrm{H}), 4.77(\mathrm{~d}, J=17.2 \mathrm{~Hz}, 1 \mathrm{H}), 4.06(\mathrm{dd}, J=$ $9.2,5.2 \mathrm{~Hz}, 1 \mathrm{H}), 3.14(\mathrm{dd}, J=18.8,9.6 \mathrm{~Hz}, 1 \mathrm{H}), 2.98(\mathrm{dd}, J=$ $18.8,5.2 \mathrm{~Hz}, 1 \mathrm{H}), 2.78(\mathrm{~s}, 3 \mathrm{H}), 2.34$ (s, 3H) ppm. ${ }^{13} \mathrm{C}\left\{{ }^{1} \mathrm{H}\right\} \mathrm{NMR}$ $\left(100 \mathrm{MHz}, \mathrm{CDCl}_{3}\right): \delta=178.2,176.4,168.5,142.0,139.2$, $137.1,133.5,132.1,131.6,130.0,129.0,128.4,124.2,122.9$, 54.2, 42.2, 36.8, 24.8, 20.9 ppm. HRMS (ESI) calcd. for [M + $\mathrm{Na}]^{+} \mathrm{C}_{20} \mathrm{H}_{18} \mathrm{~N}_{2} \mathrm{O}_{3} \mathrm{Na}$ 357.1210, found 357.1212 (1 ppm).

3-(4-Methoxy-2-(1-oxoisoindolin-2-yl)phenyl)-1-methylpyrrolidine-2,5-dione $(\mathbf{2 h})$. White solid, yield $=76 \%, 132.9 \mathrm{mg}$. Mp: 154-156 ${ }^{\circ} \mathrm{C}$. ${ }^{1} \mathrm{H}$ NMR $\left(400 \mathrm{MHz}, \mathrm{CDCl}_{3}\right): \delta=7.87(\mathrm{~d}, J=7.6$ $\mathrm{Hz}, 1 \mathrm{H}), 7.60(\mathrm{dd}, J=7.2,7.2 \mathrm{~Hz}, 1 \mathrm{H}), 7.50(\mathrm{dd}, J=7.2,6.4$ $\mathrm{Hz}, 2 \mathrm{H}), 7.12(\mathrm{~d}, J=8.4 \mathrm{~Hz}, 1 \mathrm{H}), 6.93(\mathrm{dd}, J=8.8,2.4 \mathrm{~Hz}, 1 \mathrm{H})$, $6.81(\mathrm{~d}, J=2.8 \mathrm{~Hz}, 1 \mathrm{H}), 4.88(\mathrm{~d}, J=17.2 \mathrm{~Hz}, 1 \mathrm{H}), 4.79(\mathrm{~d}, J=$ $16.8 \mathrm{~Hz}, 1 \mathrm{H}), 4.03(\mathrm{dd}, J=9.6,5.2 \mathrm{~Hz}, 1 \mathrm{H}), 3.78(\mathrm{~s}, 3 \mathrm{H}), 3.15$ $(\mathrm{dd}, J=18.8,9.6 \mathrm{~Hz}, 1 \mathrm{H}), 2.96(\mathrm{dd}, J=18.8,4.8 \mathrm{~Hz}, 1 \mathrm{H}), 2.79$ (s, 3H) ppm. ${ }^{13} \mathrm{C}\left\{{ }^{1} \mathrm{H}\right\}$ NMR $\left(100 \mathrm{MHz}, \mathrm{CDCl}_{3}\right): \delta=178.4$, $176.5,168.5,159.9,142.0,138.3,132.2,131.5,129.2,128.53$, $128.50,124.3,123.0,115.1,113.9,55.6,54.2,42.0,36.9,24.9$ ppm. HRMS (ESI) calcd. for $\left[\mathrm{M}+\mathrm{Na}^{+} \mathrm{C}_{20} \mathrm{H}_{18} \mathrm{~N}_{2} \mathrm{O}_{4} \mathrm{Na}\right.$ 373.1159, found $373.1162(1 \mathrm{ppm})$.

3-(3-Fluoro-2-(1-oxoisoindolin-2-yl)phenyl)-1-methylpyrrolidine-2,5-dione (2i). White solid, yield $=84 \%, 84.9 \mathrm{mg}$. Mp: $140-142^{\circ} \mathrm{C}$. This compound exists as a mixture (63:37) of two rotamers. ${ }^{1} \mathrm{H}$ NMR $\left(400 \mathrm{MHz}, \mathrm{CDCl}_{3}\right): \delta=7.89(\mathrm{~d}, J=7.6 \mathrm{~Hz}$, $1 \mathrm{H}$, major), $7.83(\mathrm{~d}, J=7.6 \mathrm{~Hz}, 1 \mathrm{H}$, minor), 7.62-7.57 (m, $1 \mathrm{H}$, major), 7.62-7.57 (m, 1H, minor), 7.52-7.45 (m, 2H, major), 7.52-7.45 (m, 2H, minor), 7.40-7.33 (m, 1H, major), 7.40-7.33 (m, 1H, minor), 7.18-7.13 (m, 1H, major), 7.18-7.13 (m, 2H, minor), $6.96(\mathrm{~d}, J=7.6 \mathrm{~Hz}, 1 \mathrm{H}$, major), $4.93(\mathrm{~d}, J=16.8 \mathrm{~Hz}$, $1 \mathrm{H}$, major), 4.85 (d, $J=16.8 \mathrm{~Hz}, 1 \mathrm{H}$, major), $4.70(\mathrm{~d}, J=16.8$ $\mathrm{Hz}, 1 \mathrm{H}$, minor), 4.50 (d, $J=16.8 \mathrm{~Hz}, 1 \mathrm{H}$, minor), 4.14-4.09 (m, $1 \mathrm{H}$, major), 4.14-4.09 (m, 1H, minor), 3.32-3.22 (m, 1H, major), 3.32-3.22 (m, 1H, minor), 3.05-2.93 (m, 1H, minor), 2.97 (s, 3H, major), 2.91-2.85 (m, 1H, major), 2.39 (s, 3H, minor) ppm. ${ }^{13} \mathrm{C}\left\{{ }^{1} \mathrm{H}\right\}$ NMR $\left(100 \mathrm{MHz}, \mathrm{CDCl}_{3}\right): \delta=177.5,177.4$, $176.2,175.5,169.4,168.6,159.9\left(\mathrm{~d}, J_{\mathrm{C}-\mathrm{F}}=248.9 \mathrm{~Hz}\right), 159.2(\mathrm{~d}$, $\left.J_{\mathrm{C}-\mathrm{F}}=249.6 \mathrm{~Hz}\right), 142.6,142.5,140.2,139.3,132.5,132.3$, 
$131.2,130.5,130.4\left(\mathrm{~d}, J_{\mathrm{C}-\mathrm{F}}=8.8 \mathrm{~Hz}\right), 130.3\left(\mathrm{~d}, J_{\mathrm{C}-\mathrm{F}}=9.0 \mathrm{~Hz}\right)$, $128.4,128.3,127.0\left(\mathrm{~d}, J_{\mathrm{C}-\mathrm{F}}=3.4 \mathrm{~Hz}\right), 125.8\left(\mathrm{~d}, J_{\mathrm{C}-\mathrm{F}}=13.4 \mathrm{~Hz}\right)$, $124.4,124.3,124.2,123.1,123.0,122.1\left(\mathrm{~d}, J_{\mathrm{C}-\mathrm{F}}=3.5 \mathrm{~Hz}\right), 116.3$ $\left(\mathrm{d}, J_{\mathrm{C}-\mathrm{F}}=20.5 \mathrm{~Hz}\right), 116.1\left(\mathrm{~d}, J_{\mathrm{C}-\mathrm{F}}=20.3 \mathrm{~Hz}\right), 52.9\left(\mathrm{~d}, J_{\mathrm{C}-\mathrm{F}}=3.4\right.$ $\mathrm{Hz}), 51.9\left(\mathrm{~d}, J_{\mathrm{C}-\mathrm{F}}=3.6 \mathrm{~Hz}\right), 44.7\left(\mathrm{~d}, J_{\mathrm{C}-\mathrm{F}}=2.6 \mathrm{~Hz}\right), 41.3\left(\mathrm{~d}, J_{\mathrm{C}-}\right.$ $\mathrm{F}=2.3 \mathrm{~Hz}), 36.9,36.3,25.2,24.4 \mathrm{ppm} .{ }^{19} \mathrm{~F}\left\{{ }^{1} \mathrm{H}\right\}$ NMR $(376$ $\mathrm{MHz}, \mathrm{CDCl}_{3}$ ): $\delta=-118.8$ (major), -119.4 (minor) ppm. HRMS (ESI) calcd. for $[\mathrm{M}+\mathrm{Na}]^{+} \mathrm{C}_{19} \mathrm{H}_{15} \mathrm{~N}_{2} \mathrm{O}_{3} \mathrm{FNa} 361.0959$, found 361.0959 (0 ppm).

3-(2-(1-Oxoisoindolin-2-yl)phenyl)-1-phenylpyrrolidine-2,5dione (2j). Brown solid, yield $=78 \%, 149.0 \mathrm{mg}$. Mp: 246-248 ${ }^{\circ} \mathrm{C} .{ }^{1} \mathrm{H}$ NMR $\left(400 \mathrm{MHz}, \mathrm{CDCl}_{3}\right): \delta=7.93(\mathrm{~d}, J=7.6 \mathrm{~Hz}, 1 \mathrm{H})$, $7.61(\mathrm{dd}, J=7.6,7.2 \mathrm{~Hz}, 1 \mathrm{H}), 7.54(\mathrm{dd}, J=7.6,7.6 \mathrm{~Hz}, 1 \mathrm{H})$, $7.45(\mathrm{dd}, J=8.0,5.2 \mathrm{~Hz}, 3 \mathrm{H}), 7.39-7.30(\mathrm{~m}, 5 \mathrm{H}), 7.03(\mathrm{~s}, 2 \mathrm{H})$, $4.88(\mathrm{~d}, J=17.2 \mathrm{~Hz}, 1 \mathrm{H}), 4.79(\mathrm{~d}, J=16.8 \mathrm{~Hz}, 1 \mathrm{H}), 4.33(\mathrm{dd}, J$ $=7.6,7.6 \mathrm{~Hz}, 1 \mathrm{H}), 3.39-3.23(\mathrm{~m}, 2 \mathrm{H}) \mathrm{ppm} .{ }^{13} \mathrm{C}\left\{{ }^{1} \mathrm{H}\right\} \mathrm{NMR}(100$ $\left.\mathrm{MHz}, \mathrm{CDCl}_{3}\right): \delta=177.0,175.3,168.8,142.2,137.6,136.8$, $132.2,131.8,131.6,129.4,129.3,129.1,128.8,128.6,128.5$, 126.4, 124.4, 123.1, 54.3, 42.8, 36.9 ppm. HRMS (ESI) calcd. for $[\mathrm{M}+\mathrm{Na}]^{+} \mathrm{C}_{24} \mathrm{H}_{18} \mathrm{~N}_{2} \mathrm{O}_{3} \mathrm{Na} 405.1210$, found $405.1210(0$ $\mathrm{ppm})$. Single crystals suitable for $\mathrm{X}$-ray diffraction studies were obtained after slow evaporation of a concentrated solution of $\mathbf{2} \mathbf{j}$ in acetone.

1-(2-Fluorophenyl)-3-(2-(1-oxoisoindolin-2-yl)phenyl)pyrrolidine-2,5-dione $(2 \boldsymbol{k})$. White solid, yield $=76 \%, 60.9 \mathrm{mg}$. Mp: < $50{ }^{\circ} \mathrm{C}$. This compound exists as a mixture $(53: 47)$ of two rotamers. ${ }^{1} \mathrm{H}$ NMR $\left(400 \mathrm{MHz}, \mathrm{CDCl}_{3}\right): \delta=7.94(\mathrm{~d}, J=7.6 \mathrm{~Hz}, 1 \mathrm{H})$, $7.61(\mathrm{dd}, J=7.6,7.2 \mathrm{~Hz}, 1 \mathrm{H}), 7.53(\mathrm{dd}, J=7.6,7.2 \mathrm{~Hz}, 1 \mathrm{H})$, 7.48-7.26 (m, 7H), 7.18-7.10 (m, 2H), $4.88(\mathrm{~d}, J=17.2 \mathrm{~Hz}, 1 \mathrm{H})$, $4.81(\mathrm{~d}, J=17.2 \mathrm{~Hz}, 1 \mathrm{H}), 4.38(\mathrm{dd}, J=9.6,5.6 \mathrm{~Hz}, 1 \mathrm{H}), 3.41$ $(\mathrm{dd}, J=18.8,9.6 \mathrm{~Hz}, 1 \mathrm{H}), 3.25-3.19(\mathrm{~m}, 1 \mathrm{H}) \mathrm{ppm} .{ }^{13} \mathrm{C}\left\{{ }^{1} \mathrm{H}\right\}$ NMR $\left(100 \mathrm{MHz}, \mathrm{CDCl}_{3}\right): \delta=176.2,174.4,168.7,157.5\left(\mathrm{~d}, J_{\mathrm{C}-}\right.$ $\mathrm{F}=251.0 \mathrm{~Hz}), 142.2,137.7,136.8,132.2,131.8,131.0\left(\mathrm{~d}, J_{\mathrm{C}-\mathrm{F}}\right.$ $=8.0 \mathrm{~Hz}), 129.3,128.5,124.6\left(\mathrm{~d}, J_{\mathrm{C}-\mathrm{F}}=3.8 \mathrm{~Hz}\right), 124.4,123.1$, $120.0\left(\mathrm{~d}, J_{\mathrm{C}-\mathrm{F}}=13.1 \mathrm{~Hz}\right), 116.6\left(\mathrm{~d}, J_{\mathrm{C}-\mathrm{F}}=19.4 \mathrm{~Hz}\right), 54.3,42.9$, 37.4 ppm. ${ }^{19} \mathrm{~F}\left\{{ }^{1} \mathrm{H}\right\}$ NMR $\left(376 \mathrm{MHz}, \mathrm{CDCl}_{3}\right): \delta=-119.2,-119.7$ ppm. HRMS (ESI) calcd. for $[\mathrm{M}+\mathrm{Na}]^{+} \mathrm{C}_{24} \mathrm{H}_{17} \mathrm{~N}_{2} \mathrm{O}_{3} \mathrm{FNa}$ 423.1115, found 423.1117 (0 ppm).

1-(2-Bromophenyl)-3-(2-(1-oxoisoindolin-2-yl)phenyl)pyrrolidine-2,5-dione (2l). Brown solid, yield $=64 \%, 88.8 \mathrm{mg}$. Mp: $136-138^{\circ} \mathrm{C}$. This compound exists as a mixture $(57: 43)$ of two rotamers. ${ }^{1} \mathrm{H}$ NMR $\left(400 \mathrm{MHz}, \mathrm{CDCl}_{3}\right): \delta=7.94(\mathrm{dd}, J=7.2$, $6.4 \mathrm{~Hz}, 1 \mathrm{H}), 7.68-7.13(\mathrm{~m}, 11 \mathrm{H}), 4.96-4.76(\mathrm{~m}, 2 \mathrm{H}), 4.43-4.37$ $(\mathrm{m}, 1 \mathrm{H}), 3.48-3.11(\mathrm{~m}, 2 \mathrm{H}) \mathrm{ppm} .{ }^{13} \mathrm{C}\left\{{ }^{1} \mathrm{H}\right\}$ NMR $(100 \mathrm{MHz}$, $\left.\mathrm{CDCl}_{3}\right): \delta=176.10,176.07,174.5,174.4,168.9,168.4,142.3$, $142.0,137.8,137.3,136.5,136.1,133.52,133.46,132.25$, $132.16,131.70,131.66,131.61,131.4,131.1,130.9,129.9$, $129.7,129.4,129.3,129.24,129.22,128.8,128.6,128.5,128.4$, $128.3,128.2,127.9,124.4,124.2,123.2,123.1,122.3,122.2$, 54.4, 54.2, 43.6, 42.2, 37.4, 37.0 ppm. HRMS (ESI) calcd. for $[\mathrm{M}+\mathrm{Na}]^{+} \mathrm{C}_{24} \mathrm{H}_{17} \mathrm{~N}_{2} \mathrm{O}_{3}{ }^{79} \mathrm{BrNa} 483.0315$, found 483.0311 ppm).

1-(4-Nitrophenyl)-3-(2-(1-oxoisoindolin-2-yl)phenyl)pyrrolidine-2,5-dione $(\mathbf{2 m})$. Brown solid, yield $=70 \%, 59.7 \mathrm{mg}$. $\mathrm{Mp}$ : 126-128 ${ }^{\circ} \mathrm{C} .{ }^{1} \mathrm{H}$ NMR $\left(400 \mathrm{MHz}, \mathrm{CDCl}_{3}\right): \delta=8.13$ (d, $J=8.4$ $\mathrm{Hz}, 2 \mathrm{H}), 7.89(\mathrm{~d}, J=7.6 \mathrm{~Hz}, 1 \mathrm{H}), 7.61(\mathrm{dd}, J=7.6,7.2 \mathrm{~Hz}, 1 \mathrm{H})$, $7.52(\mathrm{dd}, J=7.6,7.2 \mathrm{~Hz}, 1 \mathrm{H}), 7.46-7.25(\mathrm{~m}, 7 \mathrm{H}), 4.79(\mathrm{~d}, J=$ $17.2 \mathrm{~Hz}, 2 \mathrm{H}), 4.37(\mathrm{dd}, J=8.0,7.6 \mathrm{~Hz}, 1 \mathrm{H}), 3.36(\mathrm{~d}, J=8.4 \mathrm{~Hz}$, $2 \mathrm{H}) \mathrm{ppm} .{ }^{13} \mathrm{C}\left\{{ }^{1} \mathrm{H}\right\}$ NMR $\left(100 \mathrm{MHz}, \mathrm{CDCl}_{3}\right): \delta=176.3,174.4$, $168.8,146.8,142.1,137.3,136.3,132.4,131.3,129.6,129.3$, $128.8,128.6,126.8,124.3,124.2,123.1,54.1,43.3,36.8$ ppm.
HRMS (ESI) calcd. for $[\mathrm{M}+\mathrm{Na}]^{+} \mathrm{C}_{24} \mathrm{H}_{17} \mathrm{~N}_{3} \mathrm{O}_{5} \mathrm{Na} 450.1060$, found 450.1063 (1 ppm).

1-Benzyl-3-(2-(1-oxoisoindolin-2-yl)phenyl)pyrrolidine-2,5-dione $(2 n)$. Brown solid, yield $=88 \%, 174.0 \mathrm{mg}$. Mp: $<50{ }^{\circ} \mathrm{C} .{ }^{1} \mathrm{H}$ NMR (400 MHz, $\left.\mathrm{CDCl}_{3}\right): \delta=7.90(\mathrm{~d}, J=7.6 \mathrm{~Hz}, 1 \mathrm{H}), 7.61$ $(\mathrm{dd}, J=7.6,7.2 \mathrm{~Hz}, 1 \mathrm{H}), 7.54-7.49(\mathrm{~m}, 2 \mathrm{H}), 7.42-7.23(\mathrm{~m}, 8 \mathrm{H})$, $7.13(\mathrm{~d}, J=7.2 \mathrm{~Hz}, 1 \mathrm{H}), 4.90(\mathrm{~d}, J=17.2 \mathrm{~Hz}, 1 \mathrm{H}), 4.81(\mathrm{~d}, J=$ $17.2 \mathrm{~Hz}, 1 \mathrm{H}), 4.58(\mathrm{~d}, J=14.0 \mathrm{~Hz}, 1 \mathrm{H}), 4.50(\mathrm{~d}, J=14.0 \mathrm{~Hz}$, $1 \mathrm{H}), 4.13(\mathrm{dd}, J=9.6,5.2 \mathrm{~Hz}, 1 \mathrm{H}), 3.22(\mathrm{dd}, J=18.8,9.6 \mathrm{~Hz}$, $1 \mathrm{H}), 2.96(\mathrm{dd}, J=18.8,5.2 \mathrm{~Hz}, 1 \mathrm{H}) \mathrm{ppm} .{ }^{13} \mathrm{C}\left\{{ }^{1} \mathrm{H}\right\} \mathrm{NMR}(100$ $\left.\mathrm{MHz}, \mathrm{CDCl}_{3}\right): \delta=177.6,176.0,168.5,142.0,137.5,136.8$, $135.7,132.1,131.6,129.2,129.1,128.7,128.6,128.4,128.3$, 128.0, 124.2, 123.0, 54.2, 42.6, 42.1, 37.1 ppm. HRMS (ESI) calcd. for $[\mathrm{M}+\mathrm{Na}]^{+} \mathrm{C}_{25} \mathrm{H}_{20} \mathrm{~N}_{2} \mathrm{O}_{3} \mathrm{Na} 419.1366$, found 419.1368 (0 ppm).

3-(2-(1-Oxoisoindolin-2-yl)phenyl)-1-(4-(trifluoromethyl)benzyl)pyrrolidine-2,5-dione (2o). White solid, yield $=88 \%, 81.3$ mg. Mp: $78-80{ }^{\circ} \mathrm{C} .{ }^{1} \mathrm{H}$ NMR $\left(400 \mathrm{MHz}, \mathrm{CDCl}_{3}\right): \delta=7.89(\mathrm{~d}, J$ $=7.6 \mathrm{~Hz}, 1 \mathrm{H}), 7.62(\mathrm{dd}, J=7.6,7.2 \mathrm{~Hz}, 1 \mathrm{H}), 7.54-7.50(\mathrm{~m}, 4 \mathrm{H})$, $7.43-7.35(\mathrm{~m}, 4 \mathrm{H}), 7.29(\mathrm{~d}, J=7.6 \mathrm{~Hz}, 1 \mathrm{H}), 7.14(\mathrm{~d}, J=7.2 \mathrm{~Hz}$, $1 \mathrm{H}), 4.88(\mathrm{~d}, J=16.8 \mathrm{~Hz}, 1 \mathrm{H}), 4.82(\mathrm{~d}, J=17.2 \mathrm{~Hz}, 1 \mathrm{H}), 4.62$ $(\mathrm{d}, J=14.0 \mathrm{~Hz}, 1 \mathrm{H}), 4.54(\mathrm{~d}, J=14.4 \mathrm{~Hz}, 1 \mathrm{H}), 4.17(\mathrm{dd}, J=$ 9.6, $5.2 \mathrm{~Hz}, 1 \mathrm{H}), 3.26(\mathrm{dd}, J=18.8,9.6 \mathrm{~Hz}, 1 \mathrm{H}), 3.02(\mathrm{dd}, J=$ $18.8,5.2 \mathrm{~Hz}, 1 \mathrm{H}) \mathrm{ppm} .{ }^{13} \mathrm{C}\left\{{ }^{1} \mathrm{H}\right\} \mathrm{NMR}\left(100 \mathrm{MHz}, \mathrm{CDCl}_{3}\right): \delta=$ $177.6,175.9,168.6,142.0,139.4\left(\mathrm{q}, J_{\mathrm{C}-\mathrm{F}}=1.6 \mathrm{~Hz}\right), 137.5$, $136.5,132.3,131.6,130.3\left(\mathrm{q}, J_{\mathrm{C}-\mathrm{F}}=32.2 \mathrm{~Hz}\right), 129.3\left(\mathrm{q}, J_{\mathrm{C}-\mathrm{F}}=\right.$ $1.5 \mathrm{~Hz}), 129.1,128.6,128.4,127.9,125.7$ (q, JC-F = 3.8 Hz), $124.3,124.0\left(\mathrm{q}, J_{\mathrm{C}-\mathrm{F}}=270.4 \mathrm{~Hz}\right), 123.1,54.2,42.3,42.1,37.1$ ppm. ${ }^{19} \mathrm{~F}\left\{{ }^{1} \mathrm{H}\right\}$ NMR $\left(376 \mathrm{MHz}, \mathrm{CDCl}_{3}\right): \delta=-62.6 \mathrm{ppm}$. HRMS (ESI) calcd. for $[\mathrm{M}+\mathrm{Na}]^{+} \mathrm{C}_{26} \mathrm{H}_{19} \mathrm{~N}_{2} \mathrm{O}_{3} \mathrm{~F}_{3} \mathrm{Na} 487.1240$, found 487.1239 (0 ppm).

3-(2-(1-Oxoisoindolin-2-yl)phenyl)-1-(thiophen-2-ylmethyl)pyrrolidine-2,5-dione (2p). White solid, yield $=58 \%, 70.1$ mg. Mp: $216-218^{\circ} \mathrm{C} .{ }^{1} \mathrm{H}$ NMR $\left(400 \mathrm{MHz}, \mathrm{CDCl}_{3}\right): \delta=7.89$ (d, $J=7.6 \mathrm{~Hz}, 1 \mathrm{H}), 7.62(\mathrm{dd}, J=7.6,7.2 \mathrm{~Hz}, 1 \mathrm{H}), 7.52(\mathrm{dd}, J=$ 7.6, 7.2 Hz, 2H), 7.43-7.34 (m, 2H), $7.29(\mathrm{dd}, J=7.2,1.6 \mathrm{~Hz}$, $1 \mathrm{H}), 7.18-7.12(\mathrm{~m}, 2 \mathrm{H}), 7.04(\mathrm{~d}, J=3.6 \mathrm{~Hz}, 1 \mathrm{H}), 6.88(\mathrm{dd}, J=$ $5.2,3.6 \mathrm{~Hz}, 1 \mathrm{H}), 4.90(\mathrm{~d}, J=17.2 \mathrm{~Hz}, 1 \mathrm{H}), 4.82(\mathrm{~d}, J=17.2 \mathrm{~Hz}$, $1 \mathrm{H}), 4.76-4.66(\mathrm{~m}, 2 \mathrm{H}), 4.13(\mathrm{dd}, J=9.6,5.2 \mathrm{~Hz}, 1 \mathrm{H}), 3.24(\mathrm{dd}$, $J=18.8,9.6 \mathrm{~Hz}, 1 \mathrm{H}), 2.97(\mathrm{dd}, J=18.8,4.8 \mathrm{~Hz}, 1 \mathrm{H}) \mathrm{ppm}$. ${ }^{13} \mathrm{C}\left\{{ }^{1} \mathrm{H}\right\}$ NMR $\left(100 \mathrm{MHz}, \mathrm{CDCl}_{3}\right): \delta=177.2,175.6,168.6$, $142.0,137.5,137.2,136.7,132.2,131.7,129.3,129.2,128.6$, $128.4,128.1,127.9,126.9,126.1,124.4,123.1,54.3,42.2,37.2$, $36.8 \mathrm{ppm}$. HRMS (ESI) calcd. for $[\mathrm{M}+\mathrm{Na}]^{+} \mathrm{C}_{23} \mathrm{H}_{18} \mathrm{~N}_{2} \mathrm{O}_{3} \mathrm{SNa}$ 425.0930, found 425.0931 (0 ppm).

3-(2-(1-Oxoisoindolin-2-yl)phenyl)pyrrolidine-2,5-dione (2q). Brown solid, yield $=10 \%, 14.8 \mathrm{mg}$. Mp: $<50{ }^{\circ} \mathrm{C} .{ }^{1} \mathrm{H}$ NMR (400 MHz, acetone- $\left.d_{6}\right): \delta=10.08(\mathrm{~s}, \mathrm{br}, 1 \mathrm{H}), 7.78(\mathrm{~d}, J=7.6 \mathrm{~Hz}$, $1 \mathrm{H}), 7.69-7.64(\mathrm{~m}, 2 \mathrm{H}), 7.55(\mathrm{ddd}, J=7.6,7.6,2.0 \mathrm{~Hz}, 1 \mathrm{H})$, 7.50-7.43 (m, 4H), $4.89(\mathrm{~d}, J=17.2 \mathrm{~Hz}, 2 \mathrm{H}), 4.36(\mathrm{dd}, J=9.6$, $5.6 \mathrm{~Hz}, 1 \mathrm{H}), 3.17(\mathrm{dd}, J=18.4,9.6 \mathrm{~Hz}, 1 \mathrm{H}), 2.95(\mathrm{dd}, J=18.4$, $5.6 \mathrm{~Hz}, 1 \mathrm{H}) \mathrm{ppm} .{ }^{13} \mathrm{C}\left\{{ }^{1} \mathrm{H}\right\} \mathrm{NMR}\left(100 \mathrm{MHz}\right.$, acetone- $\left.d_{6}\right): \delta=$ $178.7,176.5,168.0,142.9,138.2,138.0,131.9,131.8,128.7$, 128.6, 128.5, 128.0, 124.5, 123.5, 123.3, 53.8, 44.0, 38.0 ppm. HRMS (ESI) calcd. for $[\mathrm{M}+\mathrm{Na}]^{+} \mathrm{C}_{18} \mathrm{H}_{14} \mathrm{~N}_{2} \mathrm{O}_{3} \mathrm{Na} 329.0897$, found $329.0895(0 \mathrm{ppm})$.

1-Methyl-3-(2-(2-oxopyrrolidin-1-yl)phenyl)pyrrolidine-2,5dione $\left(\boldsymbol{\sigma}^{\prime}\right)$. White solid, yield $=80 \%, 65.2 \mathrm{mg}$. Mp: $145-147^{\circ} \mathrm{C}$. ${ }^{1} \mathrm{H}$ NMR $\left(400 \mathrm{MHz}, \mathrm{CDCl}_{3}\right): \delta=7.34-7.26(\mathrm{~m}, 2 \mathrm{H}), 7.17-7.12$ $(\mathrm{m}, 2 \mathrm{H}), 4.07(\mathrm{dd}, J=9.6,5.2 \mathrm{~Hz}, 1 \mathrm{H}), 3.83-3.75(\mathrm{~m}, 2 \mathrm{H}), 3.17$ $(\mathrm{dd}, J=18.8,9.6 \mathrm{~Hz}, 1 \mathrm{H}), 3.01(\mathrm{~s}, 3 \mathrm{H}), 2.87(\mathrm{dd}, J=18.8,4.8$ 
$\mathrm{Hz}, 1 \mathrm{H}), 2.49$ (t, $J=8.4 \mathrm{~Hz}, 2 \mathrm{H}), 2.23-2.10(\mathrm{~m}, 2 \mathrm{H}) \mathrm{ppm}$. ${ }^{13} \mathrm{C}\left\{{ }^{1} \mathrm{H}\right\}$ NMR $\left(100 \mathrm{MHz}, \mathrm{CDCl}_{3}\right): \delta=177.9,176.4,175.4$, 138.0, 135.8, 129.1, 128.7, 128.3, 127.2, 51.6, 42.4, 37.0, 31.2, 25.1, 19.0 ppm. HRMS (ESI) calcd. for $[\mathrm{M}+\mathrm{Na}]^{+}$ $\mathrm{C}_{15} \mathrm{H}_{16} \mathrm{~N}_{2} \mathrm{O}_{3} \mathrm{Na}$ 295.1053, found 295.1053 (0 ppm).

Large Scale Reaction for the Ruthenium-Catalyzed C-H bond Alkylation: $\left[\mathrm{RuCl}_{2}(p \text {-cymene })\right]_{2}(7.5 \mathrm{~mol} \%, 375 \mathrm{mg})$, $\mathrm{AgSbF}_{6}(30 \mathrm{~mol} \%, 515 \mathrm{mg}), \mathrm{Cu}(\mathrm{OAc})_{2} \cdot \mathrm{H}_{2} \mathrm{O}(7.5 \mathrm{mmol}, 1.5 \mathrm{~g}$, 1.5 equiv.), 6 ( $5 \mathrm{mmol}, 806 \mathrm{mg}, 1.0$ equiv.), and $N$-methylmaleimide ( $10 \mathrm{mmol}, 1.111 \mathrm{~g}, 2.0$ equiv.) were taken in a $100 \mathrm{~mL}$ pressure tube, which was equipped with a magnetic stirrer. To this mixture was added $\mathrm{AcOH}(25 \mathrm{mmol}, 1.5 \mathrm{~mL}, 5.0$ equiv.) and solvent DCE $(25 \mathrm{~mL})$ under Argon. Then the reaction vial was sealed with the screw cap and allowed to stir at $120^{\circ} \mathrm{C}$ in an oil bath for $18 \mathrm{~h}$. After being cooled to ambient temperature, the reaction mixture was diluted with $\mathrm{CH}_{2} \mathrm{Cl}_{2}$ and then filtered through Celite. After evaporation of the solvent in vacuo, the crude product was purified by column chromatography on silica gel ( $n$-heptane/EtOAc: $1: 1$ to $2: 1)$ to give the desired product 6 ' $(1.12 \mathrm{~g}, 85 \%$ yield $)$

\section{ASSOCIATED CONTENT}

\section{Supporting Information}

The Supporting Information is available free of charge on the ACS Publications website.

Deuteration experiments, and NMR spectra for all new compounds (PDF)

Crystallographic data for compound $\mathbf{2 j}$ (CIF)

\section{AUTHOR INFORMATION}

\section{Corresponding Author}

* E-mail: rafael.gramage-doria@univ-rennes1.fr

\section{ACKNOWLEDGMENT}

CNRS, University of Rennes 1, Rennes Metropole, China Scholarship Council (PhD grant to Y.-C.Y.), Agence Nationale de la Recherche (ANR-JCJC) and COST Action CA15106 (CHAOS) are acknowledged.

\section{REFERENCES}

(1) (a) Evano, G.; Theunissen, C. Beyond Friedel and Crafts: Directed Alkylation of C-H Bonds in Arenes. Angew. Chem. Int. Ed. 2019, 58, 7202-7236. (b) From C-H to C-C Bonds: Cross-Dehydrogenative-Coupling (Ed.: Li, C.-J.), 2014, RSC, Cambridge, UK. (c) Narayan, R.; Matcha, K.; Antonchick, A. P. Metal-Free Oxidative C-C Bond Formation through C-H Bond Functionalization. Chem. Eur. J. 2015, 21, 14678-14693. (d) Zhang, Y.-H.; Shi, G.-F.; Yu, J.-Q. in Comprehensive Organic Synthesis $2^{\text {nd }}$ Edition (Eds.: Knochel, P.; Molander, G. A.) 2014, Elsevier, Amsterdam, pp 1101-1209.

(2) (a) Sambiagio, C.; Schoenbauer, D.; Blieck, R.; Dao-Huy, T.; Pototschnig, G.; Schaaf, P.; Wiesinger, T.; Zia, M. F.; Wencel-Delord, J.; Besset, T.; Maes, B. U. W.; Schnuerch, M. A Comprehensive Overview of Directing Groups Applied in Metal-Catalyzed C-H Functionalization Chemistry. Chem. Soc. Rev. 2018, 47, 6603-6743. (b) Dhakshinamoorthy, A.; Asiri, A. B.; Garcia, H. Formation of C-C and CHeteroatom Bonds by C-H Activation by Metal Organic Frameworks as Catalysts or Supports. ACS Catal. 2019, 9, 1081-1102. (c) Santoro, S.; Kozhushkov, S. I.; Ackermann, L.; Vaccaro, L. Heterogeneous Catalytic Approaches in C-H Activation Reactions. Green Chem. 2016, 18, 3471-3493. (d) Yamaguchi, J.; Yamaguchi, A. D.; Itami, K. C-H Bond Functionalization: Emerging Synthetic Tools for Natural Products and Pharmaceuticals. Angew. Chem. Int. Ed. 2012, 51, 8960-9009.
(3) Murai, S.; Kakiuchi, F.; Sekine, S.; Tanako, Y.; Kamatani, A.; Sonoda, M.; Chatani, N. Efficient Catalytic Addition of Aromatic Carbon-Hydrogen Bonds to Olefins. Nature 1993, 366, 529-531.

(4) (a) Catalytic Hydroarylation of Carbon-Carbon Multiple Bonds (Ed.: Ackermann, L.) 2018, Wiley-VCH, Weinheim, Germany. (b) Hussain, I.; Singh, T. Synthesis of Biaryls through Aromatic C-H Bond Activation. A Review of Recent Developments. Adv. Synth. Catal. 2014, 356, 1661-1696. (c) Colby, D. A.; Bergman, R. G.; Ellman, J. A. Rhodium-Catalyzed C-C Bond Formation via Heteroatom-Directed CH Bond Activation. Chem. Rev. 2010, 110, 624-655. (d) Seregin, I. V.; Gevorgyan, V. Direct Transition Metal-Catalyzed Functionalization of Heteroaromatic Compounds. Chem. Soc. Rev. 2007, 36, 1173-1193. (e) Kozhushkov, S. I.; Ackermann, L. Ruthenium-Catalyzed Direct Oxidative Alkenylation of Arenes through Twofold C-H Bond Functionalization. Chem. Sci. 2013, 4, 886-896. (f) Zhang, F.; Spring, D. A. Arene C-H Functionalisation Using a Removable/Modifiable or a Traceless Directing Group Strategy. Chem. Soc. Rev. 2014, 43, 6906-6919. (g) Alberico, D.; Scott, M. E.; Lautens, M. Aryl-Aryl Bond Formation by Transition-Metal-Catalyzed Direct Arylation. Chem. Rev. 2007, 107, 174-238. (h) Daugulis, O.; Do, H.-Q.; Shabashov, D. Palladium- and Copper-Catalyzed Arylation of Carbon-Hydrogen Bonds. Acc. Chem. Res. 2009, 42, 1074-1086. (i) Dong, Z.; Ren, Z.; Thompson, S. J.; Xu, Y.; Dong, G. Transition-Metal-Catalyzed C-H Alkylation Using Alkenes. Chem. Rev. 2017, 117, 9333-9403.

(5) (a) Zhu, C.; Falck, J. R. Rhodium Catalyzed C-H Olefination of $N$-Benzoylsulfonamides with Internal Alkenes. Chem. Commun. 2012, 48, 1674-1676. (b) Miura, W.; Hirano, K.; Miura, M. Copper-Mediated Oxidative Coupling of Benzamides with Maleimides via Directed C-H Cleavage. Org. Lett. 2015, 17, 4034-4037. (c) Bettadapur, K. R.; Lanke, V.; Prabhu, K. R. Ru (II)-Catalyzed C-H Activation: KetoneDirected Novel 1,4-Addition of Ortho C-H Bond to Maleimides. Org. Lett. 2015, 17, 4658-4661. (d) Lanke, V.; Bettadapur, K. R.; Prabhu, K. R. Site-Selective Addition of Maleimide to Indole at the C-2 Position: Ru(II)-Catalyzed C-H Activation. Org. Lett. 2015, 17, 4662-4665. (e) Sharma, S.; Han, S. H.; Oh, Y. Mishra, N. K.; lee, S. H.; Oh, J. S.; Kim, I. S. Cross-Coupling of Acrylamides and Maleimides under Rhodium Catalysis: Controlled Olefin Migration. Org. Lett. 2016, 18, 2568-2571. (f) Han, S.; Park, J.; Kim, S.; Lee, S. H.; Sharma, S.; Mishra, N. K.; Jung, Y. H.; Kim, I. S. Rhodium(III)-Catalyzed C( $\left.\mathrm{sp}^{3}\right)-$ H Alkylation of 8-Methylquinolines with Maleimides. Org. Lett. 2016, 18, 4666-4669. (g) Keshri, P.; Bettadapur, K. R.; Lanke, V.; Prabhu, K. R. Ru(II)-Catalyzed C-H Activation: Amide-Directed 1,4-Addition of the Ortho C-H Bond to Maleimides. J. Org. Chem. 2016, 81, 60566065. (h) Sharma, S.; Oh, Y.; Mishra, N. K.; De. U.; Jo, H.; Sachan, R.; Kim, H. S.; Jung, Y. H.; Kim, I. S. Rhodium-Catalyzed [3+2] Annulation of Cyclic $N$-Acyl Ketimines with Activated Olefins: Anticancer Activity of Spiroisoindolinones. J. Org. Chem. 2017, 82, 33593367. (i) Muniraj, N.; Prabhu, K. R. Cobalt(III)-Catalyzed C-H Activation: Azo Directed Selective 1,4-Addition of Ortho C-H Bond to Maleimides. J. Org. Chem. 2017, 82, 6913-6921. (j) Manoharan, R.; Jeganmohan, M. Cobalt-Catalyzed Oxidative Cyclization of Benzamides with Maleimides: Synthesis of Isoindolone Spirosuccinimides. Org. Lett. 2017, 19, 5884-5887. (k) Zhang, Z.; Han, S.; Tang, M.; Ackermann, L.; Li, J. C-H Alkylations of (Hetero)Arenes by Maleimides and Maleate Esters through Cobalt(III) Catalysis. Org. Lett. 2017, 19, 3315-3318. (1) Liu, S. L.; Li, Y.; Guo, J.-R.; Yang, G.-C.; Li, X.-H.; Gong, J.-F.; Song, M.-P. An Approach to 3-(Indol-2-yl)succinimide Derivatives by Manganese-Catalyzed C-H Activation. Org. Lett. 2017, 19, 4042-4045. (m) Mandal, A.; Sahoo, H.; Dana, S.; Baidya, M. Ruthenium(II)-Catalyzed Hydroarylation of Maleimides Using Carboxylic Acids as a Traceless Directing Group. Org. Lett. 2017, 19, 41384141. (n) Bettadapur, K. R.; Lanke, V.; Prabhu, K. R. A Deciduous Directing Group Approach for the Addition of Aryl and Vinyl Nucleophiles to Maleimides. Chem. Commun. 2017, 53, 6251-6254. (o) Han, S. H.; Mishra, N. K.; Jo, H.; Oh, Y.; Jeon, M.; Kim, S.; Kim, W. J.; Lee, J. S.; Kim, H. S.; Kim, I. S. One-pot Synthesis of Oxindoles through C-H Alkylation and Intramolecular Cyclization of Azobenzenes with Internal Olefins. Adv. Synth. Catal. 2017, 359, 2396-2401. (p) Han, S. H.; Mishra, N. K.; Jeon, M.; Kim, S.; Kim, H. S.; Jung, S.Y.; Jung, Y.-H.; Ku, J.-M.; Kim, I. S. Rhodium(III)-Catalyzed Diastereoselective Synthesis of 1-Aminoindanes via C-H Activation. Adv. 
Synth. Catal. 2017, 359, 3900-3904. (q) Zhao, H.; Shao, X.; Wang, T.; Zhai, S.; Qiu, S.; Tao, C.; Wang, H.; Zhai, H. A 2-(1-Methylhydrazinyl)pyridine-Directed $\mathrm{C}-\mathrm{H}$ Functionalization/Spirocyclization Cascade: Facile Access to Spirosuccinimide Derivatives. Chem. Commun. 2018, 54, 4927-4930, and references cited therein.

(6) (a) Sharma, S.; Han, S. H.; Jo, H.; Han, S.; Mishra, N. K.; Choi, M.; Jeong, T.; Park, J.; Kim, I. S. Rhodium-Catalyzed Vinylic C-H Functionalization of Enol Carbamates with Maleimides. Eur. J. Org. Chem. 2016, 3611-3618. (b) Yu, W.; Zhang, W.; Liu, Y.; Liu, Z.; Zhang, Y. Cobalt(III)-Catalyzed Cross-Coupling of Enamides with Allyl acetates/Maleimides. Org. Chem. Front. 2017, 4, 77-80. (c) Sherikar, M. S.; Kapanaiah, R.; Lanke, V.; Prabhu, K. R. Rhodium(III)Catalyzed C-H Activation at the C4-Position of Indole: Switchable Hydroarylation and Oxidative Heck-Type Reactions of Maleimides. Chem. Commun. 2018, 54, 11200-11203. (d) Tamizmani, M.; Gouranga, N.; Jeganmohan, M. Rhodium(III)-Catalyzed orthoAlkenylation of Anilides with Maleimides. ChemistrySelect 2019, 4, 2976-2981. (e) Pan, C.; Wang, Y.; Wu, C.; Yu, J.-T. Rhodium-Catalyzed C7-Alkylation of Indolines with Maleimides. Org. Biomol. Chem. 2018, 16, 693-697. (f) For an example with a cobalt catalyst with a very specific type of substrates, see: Banjare, S. K.; Chebolu, R.; Ravikumar, P. C. Cobalt Catalyzed Hydroarylation of Michael Acceptors with Indolines Directed by a Weakly Coordinating Functional Group. Org. Lett. 2019, 21, 4049-4053.

(7) (a) Zhan, B.-B.; Li, Y.; Xu, J.-W.; Nie, X.-L.; Fan, J.; Jin, L.; Shi, B.-F. Site-Selective $\delta$-C $\left(\mathrm{sp}^{3}\right)$-H Alkylation of Amino Acids and Peptides with Maleimides via a Six-Membered Palladacycle. Angew. Chem. Int. Ed. 2018, 57, 5858-5862. (b) Katritzky, A. R.; Yao, J.; Qi, M.; Chou, Y.; Sikora, D. J.; Davis, S. Ring Opening Reactions of Succinimides. Heterocycles 1998, 48, 2677-2691. (c) Shemchuk, L.; Chernykh, V.; Arzumanov, P.; Levashov, D.; Shemchuk, L. Synthesis of $N$ (4-oxo-3,4-dihydroquinazolin-3-yl)succinimide and $N$-(4-oxoquinazolin-3-yl)succinamic Acid Derivatives Based Thereon. Russ. J. Org. Chem. 2007, 43, 615-618. (d) Gali, H.; Prabhu, K. R.; Karra, S. R.; Katti, K. V. Facile Ring-Opening Reactions of Phthalimides as a New Strategy to Synthesize Amide-Functionalized Phosphonates, Primary Phosphines, and Bisphosphines. J. Org. Chem. 2000, 65, 676679.

(8) Manoharan, R.; Jeganmohan, M. Alkylation, Annulation, and Alkenylation of Organic Molecules with Maleimides by TransitionMetal-Catalyzed C-H Bond Activation. Asian J. Org. Chem. 2019, 8, 1949-1969.

(9) (a) Ackermann, L.;Diers, E.; Manvar, A. Ruthenium-Catalyzed C-H Bond Arylations of Arenes Bearing Removable Directing Groups via Six-Membered Ruthenacycles. Org. Lett. 2012, 14, 1154-1157. (b) Ma, W.; Ackermann, L. Ruthenium(II)-Catalyzed C-H Alkenylations of Phenols with Removable Directing Groups. Chem. Eur. J. 2013, 19, 13925-13928. (c) Nakanowatari, S.; Ackermann, L. Ruthenium(II)Catalyzed C-H Functionalizations with Allenes: Versatile Allenylations and Allylations. Chem. Eur. J. 2015, 21, 16246-16251. (d) Bruneau, C.; Gramage-Doria, R. One-Pot Directing Group Formation/C-H Bond Functionalization via Copper(I) and Ruthenium(II) Catalysis. Adv. Synth. Catal. 2016, 358, 3847-3856. (e) Ackermann, L.; Wang, L.; Wolfram, R.; Lygin, A. V. Ruthenium-Catalyzed Oxidative C-H Alkenylations of Anilides and Benzamides in Water. Org. Lett. 2012, 14, 728-731. (f) Li, J.; Kornhaass, C.; Ackermann, L. Ruthenium-Catalyzed Oxidative C-H Alkenylation of Aryl Carbamates. Chem. Commun. 2012, 48, 11343-11345. (g) Chidipudi, S. R.; Wieczysty, M. D.; Khan, I.; Lam, H. W. Synthesis of Benzopyrans by Pd(II)- or Ru(II)Catalyzed C-H Alkenylation of 2-Aryl-3-hydroxy-2-cyclohexenones. Org. Lett. 2013, 15, 570-573. (h) Manikandan, R.; Jeganmohan, M. Ruthenium-Catalyzed Cyclization of Anilides with Substituted Propiolates or Acrylates: An Efficient Route to 2-Quinolinones. Org. Lett. 2014, 16, 3568-3571. (i) Leitch, J. A.; Wilson, P. B.; McMullin, C. L.; Mahon, M. F.; Bhonoah, Y.; Williams, I. H.; Frost, C. G. Ruthenium(II) -Catalyzed C-H Functionalization Using the Oxazolidinone Heterocycle as a Weakly Coordinating Directing Group: Experimental and Computational Insights. ACS Catal. 2016, 6, 5520-5529. (j) Fabry, D. C.; Ronge, M. A.; Zoller, J.; Rueping, M. C-H Functionalization of Phenols using Combined Ruthenium and Photoredox Catalysis: In Situ Generation of the Oxidant. Angew. Chem. Int. Ed. 2015, 54, 2801-2805.
(1) Bechtoldt, A.; Tirler, C.; Raghuvanshi, K.; Warratz, S.; Kornhaass, C.; Ackermann, L. Ruthenium Oxidase Catalysis for Site-Selective C$\mathrm{H}$ Alkenylations with Ambient $\mathrm{O}_{2}$ as the Sole Oxidant. Angew. Chem. Int. Ed. 2016, 55, 264-267. (1) Li, T.; Zhang, J.; Yu, C.; Lu, X.; Xu, L.; Zhong, G. Ruthenium-Catalyzed Olefinic C-H Alkenylation of EnolCarbamates: Highly Stereo-Selective Synthesis of $(Z, Z)$ and $(Z, E)$-Butadienes. Chem. Commun. 2017, 53, 12926-12929. (m) Suzuki, C.; Hirano, K.; Satoh, T.; Miura, M. Ruthenium-Catalyzed Regioselective C-H Alkenylation Directed by a Free Amino Group. Org. Lett. 2013, 15, 3990-3993, and references cited therein.

(10) (a) Speck, K.; Magauer, T. The Chemistry of Isoindole Natural Products. Beilstein J. Org. Chem. 2013, 9, 2048-2078. (b) Bhatia, R. K. Isoindole Derivatives: Propitious Anticancer Structural Motifs. Curr. Top. Med. Chem. 2017, 17, 189-207. (c) Lunn, M. R.; Root, D. E.; Martino, A. M.; Flaherty, S. P.; Kelley, B. P.; Coovert, D. D.; Burghes, A. H.; Man, N. T.; Morris, G. E.; Zhou, J.; Androphy, E. J.; Sumner, C. J. Stockwell, B. R. Indoprofen Upregulates the Survival Motor Neuron Protein through a Cyclooxygenase-Independent Mechanism. Chem. Biol. 2004, 11, 1489-1493. (d) Miyachi, H.; Azuma, A.; Hioki, E.; Kobayashi, Y.; Iwasaki, S.; Hashimoto, Y. Inducer-Specific Regulators of Tumor Necrosis Factor Alpha Production. Chem. Pharm. Bull. 1996, 44, 1980-1982. (e) Park, J. S.; Moon, S. C.; Baik, K. U.; Cho, J. Y.; Yoo, E. S.; Byun, Y. S.; Park, M. H. Synthesis and SAR Studies for the Inhibition of TNF- $\alpha$ Production. Part 2. 2-[3-(Cyclopentyloxy)-4-methoxyphenyl]-substituted-1-isoindolinone Derivatives. Arch. Pharm. Res. 2002, 25, 137-142. (f) Hamprecht, D.; Micheli, F.; Tedesco, G.; Checchia, A.; Donati, D.; Petrone, M.; Terreni, S.; Wood, M. Isoindolone Derivatives, a New Class of 5-HT2C Antagonists: Synthesis and Biological Evaluation. Bioorg. Med. Chem. Lett. 2007, 17, 428-433. (g) Hunter, R.; Richards, P. Stereoselective Tetrapyrido[2,1-a]isoindolone Synthesis via Carbanionic and Radical Intermediates: a Model Study for the Tacaman Alkaloid D/E Ring Fusion. Org. Biomol. Chem. 2003, 1, 2348-2356. (h) Thapa, P.; Corral, E.; Sardar, S.; Pierce, B. S.; Foss Jr., F. W. Isoindolinone Synthesis: Selective Dioxane-Mediated Aerobic Oxidation of Isoindolines. J. Org. Chem. 2019, 84, 1025-1034, and references cited therein.

(11) Muniraj, S.; Prabhu, K. R. Co(III)-Catalyzed C-H Activation: A Site-Selective Conjugate Addition of Maleimide to Indole at the C2 Position. ACS Omega 2017, 2, 4470-4479.

(12) Gandeepan, P.; Mueller, T.; Zell, D.; Cera, G.; Warratz, S.; Ackermann, L. 3d Transition Metals for C-H Activation. Chem. Rev. 2019, 119, 2192-2452.

(13) (a) Phani, Kumar, N. Y.; Rogge, T.; Reddy Yetra, S.; Bechtoldt, A.; Clot, E.; Ackermann, L. Mild Decarboxylative C-H Alkylation: Computational Insights for Solvent-Robust Ruthenium(II) Domino Manifold. Chem. Eur. J. 2017, 23, 17449-17453. (b) Yu, J.-T.; Chen, R.; Jia, H.; Pan, C. Rhodium-Catalyzed Site-Selective ortho-C-H Activation: Enone Carbonyl Directed Hydroarylation of Maleimides. $J$. Org. Chem. 2018, 83, 12086-12093. (c) Li, F.; Zhou, Y.; Yang, H.; Liu, D.; Sun, B.; Zhang, F.-L. Assembly of Diverse Spirocyclic Pyrrolidines via Transient Directing Group Enabled Ortho-C $\left(\mathrm{sp}^{2}\right)-\mathrm{H}$ Alkylation of Benzaldehydes. Org. Lett. 2018, 20, 146-149.

(14) (a) Engle, K. M.; Mei, T.-S.; Wasa, M.; Yu, J.-Q. Weak Coordination as a Powerful Means for Developing Broadly Useful C-H Functionalization Reactions. Acc. Chem. Res. 2012, 45, 788-802. (b) De Sarkar, S.; Liu, W.; Kozhushkov, S. I.; Ackermann, L. Weakly Coordinating Directing Groups for Ruthenium(II)-Catalyzed C-H Activation. Adv. Synth. Catal. 2014, 356, 1461-1479. (c) Yang, Y.; Li, K.; Cheng, Y.; Wan, D.; Li, M.; You, J. Rhodium-Catalyzed Annulation of Arenes with Alkynes through Weak Chelation-Assisted C-H Activation. Chem. Commun. 2016, 52, 2872-2884. (d) Zheng, Q.-Z.; Jiao, N. Transition-Metal-Catalyzed Ketone-Directed Ortho-C-H Functionalization Reactions. Tetrahedron Lett. 2014, 55, 1121-1126. (e) Das, R.; Kumar, G. S.; Kapur, M. Amides as Weak Coordinating Groups in Proximal C-H Bond Activation. Eur. J. Org. Chem. 2017, 5439-5459. (f) da Silva Junior, E. N.; Jardim, G. A. M.; Gomes, R. S.; Liang, Y.F.; Ackermann, L. Weakly-Coordinating $N$-oxide and Carbonyl Groups for Metal-Catalyzed C-H Activation: the Case of A-Ring Functionalization. Chem. Commun. 2018, 54, 7398-7411. (g) Kang, D.; Ahn, K.; Hong, S. Site-Selective C-H Bond Functionalization of Chromones and Coumarins. Asian J. Org. Chem. 2018, 7, 1136-xx. 
(15) (a) Shan, C.; Zhu, L.; Qu, L.-B.; Bai, R.; Lan, Y. Mechanistic View of Ru-Catalyzed C-H Bond Activation and Functionalization: Computational Advances. Chem. Soc. Rev. 2018, 47, 7552-7576. (b) Ackermann, L. Carboxylate-Assisted Transition-Metal-Catalyzed C-H Bond Functionalizations: Mechanism and Scope. Chem. Rev. 2011, 111, 1315-1345. (c) Ferre Flegeau, E.; Bruneau, C.; Dixneuf, P. H.; Jutand, A. Autocatalysis for C-H Bond Activation by Ruthenium(II) Complexes in Catalytic Arylation of Functional Arenes. J. Am. Chem. Soc. 2011, 133, 10161-10170. (d) Davies, D. L.; MacGregor, S. A.; McMullin, C. L. Computational Studies of Carboxylate-Assisted C-H Activation and Functionalization at Group 8-10 Transition Metal Centers. Chem. Rev. 2017, 117, 8649-8709. (e) Fabre, I.; von Wolff, N.; Le Duc, G.; Ferrer Flegeau, E.; Bruneau, C.; Dixneuf, P. H.; Jutand, Autocatalytic Intermolecular versus Intramolecular Deprotonation in $\mathrm{C}-\mathrm{H}$ Bond Activation of Functionalized Arenes by Ruthenium(II) or Palladium(II) Complexes. Chem. Eur. J. 2013, 19, 7595-7604.

(16) (a) Yuan, Y.-C.; Bruneau, C.; Roisnel, T.; Gramage-Doria, R. Ru-Catalyzed Selective C-H Bond Hydroxylation of Cyclic Imides. $J$. Org. Chem. 2019, 84, 1898-18907. (b) Yuan, Y.-C.; Bruneau, C.; Roisnel, T.; Gramage-Doria, R. Site-selective Ru-catalyzed C-H bond alkenylation with biologically relevant isoindolinones: a case of catalyst performance controlled by subtle stereo-electronic effects of the weak directing group. Catal. Sci. Technol. 2019, 9, 4711-4717. (c) Y Yuan, Y.-C.; Bruneau, C.; Roisnel, T.; Gramage-Doria, R. Site-Selective Ruthenium-Catalyzed C-H Bond Arylations with Boronic Acids:
Exploiting Isoindolinones as a Weak Directing Group. J. Org. Chem. 2019, 84, 12893-12903.

(17) Yuan, Y.-C.; Bruneau, C.; Roisnel, T.; Gramage-Doria, R. Ruthenium(II)-catalysed selective $\mathrm{C}\left(\mathrm{sp}^{2}\right)-\mathrm{H}$ bond benzoxylation of biologically appealing $\mathrm{N}$-arylisoindolinones. Org. Biomol. Chem. 2019, $17,7517-7525$.

(18) (a) Zhou, Y.; Chen, P.; Lv, X.; Niu, J.; Wang, Y.; Lei, M.; Hu, L. A facile and efficient method for the synthesis of $\mathrm{N}$-substituted isoindolin-1-one derivatives under $\mathrm{Pd}(\mathrm{OAc})_{2} / \mathrm{HCOOH}$ system. Tet. Lett. 2017, 58, 2232-2235. (b) Kim, E.; Jeong, M.; Lee, B. M.; Kim, B. H. One-pot synthesis of isoindolin-1-ones from nitroarenes and $o$ phthalaldehyde via indium-mediated reductive condensation reaction. Heterocycles 2018, 96, 1759-1770.

(19) Lin, C.; Zhen, L.; Cheng, Y.; Du, H.-J.; Zhao, H.; Wen, X.; Kong, L.-Y.; Xu, Q.-L.; Sun, H. Visible Light-Induced Isoindole Formation To Trigger Intermolecular Diels-Alder Reactions in the Presence of Air. Org. Lett. 2015, 17, 2684-2687.

(20) Hsieh, J.-C.; Cheng, C.-H. Nickel-catalyzed coupling of isocyanates with 1,3-iodo esters and halobenzenes: a novel method for the synthesis of imide and amide derivatives. Chem. Commun. 2005, 36, 4554-4556.

(21) Kaminski, K.; Wiklik, B.; Obniska, Synthesis, Anticonvulsant Properties, and SAR Analysis of Differently Substituted Pyrrolidine-2, 5-diones and Piperidine-2,6-diones. J. Arch. Pharm. Chem. Life Sci. 2014, $347,840-852$ 